\title{
Mutual positions of the Galilean satellites of Jupiter from photometric observations during their mutual occultations and eclipses in 1997
}

N.V. Emelianov ${ }^{1}$, A.A. Berejnoi ${ }^{2}$, S.N. Vashkovjak ${ }^{1}$, E.A. Glushkova ${ }^{3}$, S.Yu. Gorda ${ }^{4}$, A. Delets $^{5}$, T.N. Dorokhova $^{6}$, N.I. Dorokhov ${ }^{6}$, V.F. Esipov ${ }^{1}$, I.S. Izmailov ${ }^{7}$, T.R. Irsmambetova ${ }^{1}$, A.A. Kiselev ${ }^{7}$, T.P. Kiseleva ${ }^{7}$, V.G. Kornilov ${ }^{1}$, V. Kucherov $^{5}$, I. Ledovskaya ${ }^{5}$, S. Mukhamednazarov ${ }^{6}$, V.N. Raskhozhev $^{2}$, V.G. Tejfel ${ }^{3}$, and G.A. Charitonova ${ }^{3}$

1 Sternberg Astronomical Institute, 13 Universitetskij prospect, 119899 Moscow, Russia e-mail: emelia@sai.msu.su

2 Observatory of Voronezh State University, Voronezh, Russia

3 Fesenkov Astrophysical Institute, Kamenskoye Plato, 480068 Almaty, Kazakhstan e-mail: tejf@afi.academ.alma-ata.su

4 Kourovka observatory of Ural state university, Ekaterinburg, Russia e-mail: Stanislav.Gorda@usu.ru

5 Main Astronomical Observatory of the Ukrainian Academy of Science, Kiev, Ukraine e-mail: leda@mao.kiev.ua

${ }^{6}$ Odessa State University, Odessa, Ukraine e-mail: tnd@pulse.tenet.odessa.ua

7 Main Astronomical Observatory, Pulkovo, 196140 St.-Petersburg, Russia e-mail: aak@pulvz.spb.su

Received July 1; accepted September 29, 1999

\begin{abstract}
We report the final results of the 1997 campaign of photometric observations of the mutual phenomena of the Galilean satellites carried out at observatories in Kazakhstan, Russia, and Ukraine. Our results contribute substantially to the world data bank of such observations and will allow the model of the motion of Galilean satellites to be further refined. To facilitate the use of photometric data, we reduced them by computing the planetocentric rectangular coordinate differences of satellite pairs for a number of instants of time so we deduce the differences for one instant from one observed light curve. It is these reduced data that constitute the principal result of this work. We based our data reduction on the method which we developed in earlier papers (Emel'yanov 1999; Emel'yanov 2000). The accuracy of observations was estimated in the process of reduction. The paper also describes the equipment used.
\end{abstract}

Key words: planets and satellites: Jupiter — techniques: photometric

Send offprint requests to: N.V. Emelianov

\section{Introduction}

Observations of mutual eclipses and occultations of planetary satellites are a source of valuable data on the dynamics of the satellites. Present-day ground-based facilities make it possible to observe mutual occultations and eclipses of the Galilean satellites of Jupiter as well as major Saturnian and Uranian satellites.

For the sake of brevity, mutual occultations and eclipses in the systems of planetary satellites are referred to as mutual phenomena. These rare events occur during about six-month long seasons separated by half the orbital period of the corresponding planet. About 300 mutual phenomena occur during each such season. Most of the mutual phenomena last from 2 to 10 minutes. What makes observations of mutual phenomena valuable is the high accuracy of astrometric data derived from the satellite photometry obtained. The data inferred from such observations are several tens to hundreds of times more accurate than the results of usual direct measurements of satellite positions.

Many observatories throughout the world observe on a regular manner mutual phenomena of planetary satellites, and both the data amount and the accuracy of satellite photometry is steadily increasing. Beginning in 1973, regular international campaigns were organized with 
the aim to observe mutual phenomena of the Galilean satellites in accordance with observing seasons. The description of specific features of mutual phenomena of planetary satellites and brief reviews of campaigns conducted can be found in Aksnes et al. (1984), Arlot (1984), Arlot et al. (1992), Nasonova (1996). Only a few researchers from the former Soviet Union had experience in performing such observations. In 1985 two light curves of the Galilean satellites were obtained at the observatory of the Fesenkov Astrophysical Institute of the Academy of Sciences of the Republic of Kazakhstan (FAI AS RK) in Almaty (Grigorjeva et al. 1986a; Grigorjeva et al. 1986b). Two observatories of CIS countries - the Crimean laboratory of the Sternberg Astronomical Institute (CL SAI) and the observatory of FAI AS RK - took part in the last campaign of observations of Saturnian satellites. A total of four light curves of the mutual phenomena of Saturnian satellites have been obtained. The techniques used and the results of astrometric reduction of satellite photometry have been published by Emel'yanov et al. (1997). This was our first experience in reducing such observations.

Seven observatories in Russia, Ukraine, and Kazakhstan took part in the next observing campaign of mutual phenomena of the Galilean satellites in 1997. Such observations have also been performed at many observatories throughout the world during this season. A large amount of satellite photometry has been obtained. These observations proved to be more accurate than those of Saturnian satellites with the Galilean satellites observed at a smaller geocentric distance and being brighter than the Saturnian ones. All this made it necessary to refine the photometric model of mutual phenomena of planetary satellites and develop more sophisticated methods for extracting positional information from such observations. Theoretical issues and the development of the tools used to construct the models of mutual phenomena of natural planetary satellites have been addressed by Emel'yanov (1999) and Emel'yanov (2000).

During the 1997 observing season of mutual phenomena of the Galilean satellites more than 40 sets of successful photometric observations have been obtained at seven observatories in Russia, Ukraine, and Kazakhstan, yielding the same number of satellite light curves.

New series of photometric satellite observations are required to refine the theory of satellite motion. This process involves the use of a huge amount of satellite photometry interpreted in terms of a model of mutual occultations and eclipses as observed from ground-based observatories, theory of planetocentric motion of satellites, and the theory of motion of planets. In this paper we suggest and apply a special technique for reduction of photometric observations of mutual phenomena of natural planetary satellites. To simplify the process of refinement of satellite orbits, we subdivide it into two stages. The input data at the first stage are the results of photometric measurements and parameters describing the observer-satellites-planet-Sun configuration. The output, after reduction of each light curve, has the form of differences of planetocentric rectangular satellite coordinates referred to a single instant of time. At the second stage the elements of satellite orbits are refined using a series of satellite coordinate differences inferred by reducing the observations all mutual phenomena carried out at different observatories.

The advantage of this approach for a user of the observational data consists of the fact that one will have a deal with a planetocentric satellite motion only to fit the satellite orbit to observations. On the other hand these data keep the whole observational reality including the observational errors.

We describe the observations made and the results of their reduction, i.e., the mutual planetocentric positions of the Galilean satellites as inferred from photometry of their mutual phenomena. Reduction of photometric observations yields to satellite position data that can be used, combined with the results of other observations, to refine the elements of satellite orbits.

\section{Description of photometric observations}

Below we give the full names of observatories where observations were made. The abbreviations of the observatory names, which we use hereafter for the sake of brevity, are printed in boldface.

- Alma-Ata. Observatory of the Fesenkov Astrophysical Institute of the Academy of Sciences of the Republic of Kazakhstan, Kazakhstan. Located in Almaty;

- Voronezh. Observatory of Voronezh State University, Voronezh, Russia. Located in Voronezh;

- Dushak. Observing station of the Astronomical Observatory of the Odessa State University, Odessa, Ukraine. Located on Mt. Dushak-Erekdag in Turkmenistan;

- Kiev. Main Astronomical Observatory of the Ukrainian Academy of Science, Kiev, Ukraine. Located in Golosyivo near Kyiv;

- Kourovka. Kourovka observatory of Ural state university, Ekaterinburg, Russia. Located in Kourovka near Ekaterinburg;

- Nauchny. Observatory of the Crimean Laboratory of the Sternberg Astronomical Institute, Moscow State University, Moscow, Russia. Located in settlement Nauchnyi, Crimea Ukraine;

- Pulkovo. Main Astronomical Observatory of the Russian Academy of Sciences, S.-Petersburg, Russia. Located in Pulkovo.

Table 1 below lists the observers who took part in observations at each observatory and a brief description of the equipment used. A separate column gives the adopted codes used of actual observers. These codes will then be 
used to identify the observatory, observers, and equipment for each observation. The observations were made with different instruments at some observatories and therefore the latter are listed twice in Table 1.

Remark in Table 1: One of the photoelectric observations at the observatory in Nauchnyi settlement was made with the participation of S.Yu. Shugarov, observer from the Sternberg Astronomical Institute.

The observations at the observatories Dushak, Kiev, Kourovka, Nauchny (Code t) were made with an electrophotometer in a photon-counting mode.

We observed mutual phenomena of the four Galilean satellites. Table 2 lists some of the parameters of the satellites and the elements of their orbits at the time close to the Jupiter opposition.

The season of mutual phenomena of Jovian satellites at observatories in Russia, Ukraine, and Kazakhstan started on March 12, 1997 and ended on January 26, 1998.

During the season of the mutual occultations and eclipses the Jupiter declination varied from -14 to -18 degrees.

\section{The technique for reduction of photometric observations of mutual phenomena of satellites}

We first consider the adopted model of mutual occultations and eclipses of natural planetary satellites as observed from the Earth. A detailed description of this model can be found in Emel'yanov (1999). Concerning the satellites they were considered as spheres with uniform surfaces.

We refer to the occulting or eclipsing satellite as the active satellite using subscript a with all quantities that refer to this satellite. We further refer to the occulted or eclipsed satellite as the passive satellite and associate subscript $\mathrm{p}$ with it.

In the case of mutual occultation at observing time $t_{0}$ the detector receives the light reflected from the passive satellite at time $t_{\mathrm{p}}$, and the light reflected from the active satellite at time $t_{\mathrm{a}}$, so that $t_{\mathrm{p}}<t_{\mathrm{a}}<t_{0}$. During observations of a mutual eclipse of the satellites the observer's detector receives at observing time $t_{0}$ the light that travelled in the vicinity of the active satellite at time $t_{\mathrm{a}}$ and was reflected from the passive satellite at time $t_{\mathrm{p}}$. In this case $t_{\mathrm{a}}<t_{\mathrm{p}}<t_{0}$. The differences between these three instants of time are determined by the time of light propagation between the satellites and the observer.

Hereafter we always refer to instants of time $t_{\mathrm{p}}$ and $t_{\mathrm{a}}$, which correspond to observing time $t_{0}$, because these three instants of time are connected by the propagation of the same photon between the satellites and the observer.

The theoretical position of the satellite is given by planetocentric coordinates $x, y, z$. The $(x, y)$-plane is chosen to be parallel to the Earth equator for the epoch of
J2000. The $x$-axis points to the vernal equinox of the same epoch.

We now consider a vector in space, which we refer to as the event vector for the sake of convenience. This vector starts at the observing point at time $t_{0}$ in the case of mutual occultation or at the solar center in the case of mutual eclipse, and ends at the center of the passive satellite at time $t_{\mathrm{a}}$.

Let $x_{\mathrm{a}}, y_{\mathrm{a}}, z_{\mathrm{a}}$ be the planetocentric coordinates of the active satellite at time $t_{\mathrm{a}}$, and $x_{\mathrm{p}}, y_{\mathrm{p}}, z_{\mathrm{p}}$, the planetocentric coordinates of the passive satellite at time $t_{\mathrm{p}}$. The flux from the passive satellite measured at time $t_{0}$ thus depends on $x_{\mathrm{p}}, y_{\mathrm{p}}, z_{\mathrm{p}}$ and $x_{\mathrm{a}}, y_{\mathrm{a}}, z_{\mathrm{a}}$.

Hereafter we consider the vector $\boldsymbol{R}_{\text {ap }}$ of the position of the active satellite at time $t_{\mathrm{a}}$ relative to the position of the passive satellite at time $t_{\mathrm{p}}$. The components of this vector in the coordinate system $x, y, z$ are determined by the following relations:

$\Delta_{x}=x_{\mathrm{a}}-x_{\mathrm{p}}, \quad \Delta_{y}=y_{\mathrm{a}}-y_{\mathrm{p}}, \quad \Delta_{z}=z_{\mathrm{a}}-z_{\mathrm{p}}$.

We now introduce another Cartesian coordinate system $X, Y, Z$ with the origin at the center of the passive satellite. Let us make the $Z$-axis parallel to the event vector and the $X$-axis, parallel to the $(x, y)$-plane. Let the $Y$-axis always make an acute angle with the $z$ axis and make up a right-hand side coordinate system together with the $X$ and $Z$ axes. This defines unambiguously the direction of the $X$-axis. Geometrically it will be possible on condition that the $z$-axis and $Z$-axis are not parallel. For the natural satellites of all known planets it is always true.

We now denote the coordinates of the active satellite in the $X, Y, Z$ reference frame at time $t_{\mathrm{a}}$ these are the components of the vector $\boldsymbol{R}_{\mathrm{ap}}$ as $X_{\mathrm{a}}, Y_{\mathrm{a}}, Z_{\mathrm{a}}$. It follows from Emel'yanov's (2000) results that the magnitude of the passive satellite, as measured at time $t_{0}$, depends on $X_{\mathrm{a}}, Y_{\mathrm{a}}$, and only slightly on projection $Z_{\mathrm{a}}$ of the mutual radius-vector of the two satellites on the event vector.

The components of the event vector $\boldsymbol{R}_{\text {ap }}$ in two reference frames are connected by the following evident relations

$X_{\mathrm{a}}=a_{x} \Delta_{x}+a_{y} \Delta_{y}+a_{z} \Delta_{z}$

$Y_{\mathrm{a}}=b_{x} \Delta_{x}+b_{y} \Delta_{y}+b_{z} \Delta_{z}$,

where coefficients $a_{x}, a_{y}, a_{z}, b_{x}, b_{y}, b_{z}$ depend only on the direction of the event vector. Note that coefficient $a_{z}$ is equal to zero.

While observing a mutual phenomenon of two satellites, the measuring system records some quantity $E$. It can be measured in arbitrary units inherent to the particular detector. In addition to the directly measured quantity $E$ we also introduce flux $S$ produced in the space by the eclipsed or occulting and occulted satellites. This flux passes then through the atmosphere and the telescope aperture up to the detector. The flux $S$ is measured in the units of the satellite flux when it is close to the eclipse and still lies outside the shadow of the occulting satellite 
Table 1. List of observers and parameters of the equipment

\begin{tabular}{|c|c|c|c|c|c|}
\hline Code & Observatory & $\begin{array}{c}\text { Altitude } \\
\text { above the } \\
\text { sea level, m }\end{array}$ & Instrument & Detector & Observers \\
\hline $\mathrm{a}$ & Alma-Ata & 1450 & Zeiss-600, $D=60 \mathrm{~cm}, F=12381 \mathrm{~mm}$ & CCD ST-6 & Tejfel V.G. \\
\hline $\mathrm{v}$ & Voronezh & 195 & Refractor, $D=6 \mathrm{~cm}, F=300 \mathrm{~mm}$ & Visual & Berejnoi A.A. \\
\hline $\mathrm{w}$ & Voronezh & 195 & Reflector, $D=10 \mathrm{~cm}, F=800 \mathrm{~mm}$ & Visual & $\begin{array}{l}\text { Berejnoi A.A., } \\
\text { Raskhozhev V.N. }\end{array}$ \\
\hline $\mathrm{d}$ & Dushak & 1000 & Reflector, $D=80 \mathrm{~cm}, F=11000 \mathrm{~mm}$ & Photometer & $\begin{array}{l}\text { Dorokhova T.N., } \\
\text { Dorokhov N.I., } \\
\text { Mukhamednazarov S. }\end{array}$ \\
\hline 1 & Kiev & 188 & $\mathrm{AZT}-2, D=70 \mathrm{~cm}, F=10500 \mathrm{~mm}$ & $\begin{array}{l}\text { Analog TV } \\
\text { photometer }\end{array}$ & $\begin{array}{l}\text { Delets A., Kucherov V. } \\
\text { Ledovskaya I. }\end{array}$ \\
\hline $\mathrm{g}$ & Kourovka & 200 & $\mathrm{AZT}-3, D=45 \mathrm{~cm}, F=10000 \mathrm{~mm}$ & Photometer & Gorda S.Yu. \\
\hline $\mathrm{t}$ & Nauchny & 550 & Zeiss-600, $D=60 \mathrm{~cm}, F=7500 \mathrm{~mm}$ & Photometer & Irsmambetova T.R. \\
\hline $\mathrm{e}$ & Nauchny & 550 & Refractor, $D=40 \mathrm{~cm}, F=1600 \mathrm{~mm}$ & CCD ST-8 & Esipov V.F. \\
\hline $\mathrm{k}$ & Pulkovo & 75 & Refractor, $D=60 \mathrm{~cm}, F=10400 \mathrm{~mm}$ & CCD ST-6 & $\begin{array}{l}\text { Kisselev A.A., } \\
\text { Izmailov I.S. }\end{array}$ \\
\hline
\end{tabular}

Table 2. Some of the satellite parameters for August 9, 1997

\begin{tabular}{|l|ccccc|}
\hline Satellite & Magnitude & Radius, km & Apparent radius, " & Apparent radius of the orbit, " & Orbital period, days \\
\hline Io & 4.88 & 1816 & 0.618 & 143 & 1.769138 \\
Europa & 5.15 & 1563 & 0.532 & 228 & 3.551181 \\
Ganimede & 4.47 & 2638 & 0.898 & 364 & 7.154553 \\
Callisto & 5.51 & 2410 & 0.821 & 641 & 16.68902 \\
\hline
\end{tabular}

in the case of mutual eclipse. In the case of mutual occultation the flux is measured in the units of the total flux $S$ from the occulting and occulted satellites. In both cases flux $S$ outside eclipse or occultation is equal to unity by definition. During a mutual occultation or eclipse flux $S$ decreases and this decrease depends primarily on $X_{\mathrm{a}}$ and $Y_{\mathrm{a}}$.

Dependence of $E$ on $S$ is usually unknown. First, we do not know beforehand what will be the reaction of the detector output on the satellite flux. Second, besides the satellite light, the detector is illuminated also by outside light (sky background, the light of the planet scatterred in the telescope). Finally it is difficult to do a forecast about the atmosphere transparency. The sometimes adopted photometer calibration using known sky light sources is often unreliable. We were led to suggest and adopt the following dependence of $E$ on $S$ :

$E=\left[K+Q\left(t-t_{\mathrm{b}}\right)\right] S\left(X_{\mathrm{a}}, Y_{\mathrm{a}}\right)+P+L\left(t-t_{\mathrm{b}}\right)$,

where $t$ is the time of observation; $t_{\mathrm{b}}$ some given instant of time, and $K, Q, P, L$, the empirical parameters to be inferred from photometric observations. The time $t_{\mathrm{b}}$ can be chosen arbitrarily prior to the reduction of the photometric observations. After the reduction both $K, P$ correspond to the same time $t_{\mathrm{b}}$. Function $S\left(X_{\mathrm{a}}, Y_{\mathrm{a}}\right)$ is determined by the model of mutual phenomena and the theory of satellite motion. The dependence $S$ on $X_{\mathrm{a}}, Y_{\mathrm{a}}$, has been analyzed by Thuillot \& Morando (1990), described by Emel'yanov (1995), Emel'yanov et al. (1997) and Emel'yanov (1999).

In general the parameters $K, Q, P, L$ may take into account the above mentioned effects. For example the parameter $Q$ corresponds to a linear variation of the atmosphere transparency and the parameters $P, L$ describe the light of the planet scatterred in the telescope. In practice although it is not a good idea to determine the parameters $Q, P, L$ from observations because of a great correlation between the errors. We do it in forced cases only when exclusion of the relevant effects by the method of observation is not possible.

The basic idea of the reduction of photometric measurements considered is as follows (Emel'yanov 2000). Denote the true values of $X_{\mathrm{a}}, Y_{\mathrm{a}}$ corresponding to a photometric measurement at certain time as $X^{(\mathrm{o})}, Y^{(\mathrm{o})}$. To reduce observations, we use one of the most accurate theories of satellite motion available. We denote theoretically computed values of $X_{\mathrm{a}}, Y_{\mathrm{a}}$, as $X_{\mathrm{t}}, Y_{\mathrm{t}}$, respectively. We further assume that the differences

$D_{x}=X^{(\mathrm{o})}-X_{\mathrm{t}}, \quad D_{y}=Y^{(\mathrm{o})}-Y_{\mathrm{t}}$,

vary negligibly within the same mutual occultation or eclipse. This allows us to compose for any photometric measurement made at time $t_{i}$ one conditional equation

$E_{i}=\left[K+Q\left(t_{i}-t_{\mathrm{b}}\right)\right] S\left(X_{\mathrm{t}}+D_{x}, Y_{\mathrm{t}}+D_{y}\right)+$

$$
+P+L\left(t_{i}-t_{\mathrm{b}}\right)
$$


for corrections $D_{x}, D_{y}$, where $X_{\mathrm{t}}, Y_{\mathrm{t}}$ are computed theoretically for the time of observation, $t_{i}$. The set of conditional equations composed for all photometric measurements of the same event can then be solved using the least squares method. The solution yields parameters $D_{x}, D_{y}, K, Q, L$, and $P$ providing the best fit to the observations made.

After doing this and to present the results of reduction, we choose arbitrarily one of the observing times, $t_{0}$, and compute $X^{(\mathrm{o})}, Y^{(\mathrm{o})}$ for this time using formulas

$X^{(o)}=X_{\mathrm{t}}+D_{x}, \quad Y^{(\mathrm{o})}=Y_{\mathrm{t}}+D_{y}$.

These values are the main result of reduction. $X^{(\mathrm{o})}$ and $Y^{(\mathrm{o})}$ can be used only if some other quantities considered here are known. In particular, we must know the time instants $t_{\mathrm{a}}$ and $t_{\mathrm{p}}$, corresponding to the chosen time $t_{0}$. With the reduction we save the values of $t_{\mathrm{a}}, t_{\mathrm{p}}, a_{x}, a_{y}, a_{z}, b_{x}, b_{y}, b_{z}$ computed for the chosen observing time, $t_{0}$.

\section{The use of the obtained data}

We now describe how to use the data obtained by reducing photometric observations of mutual phenomena of satellites in order to refine the theory of satellite motion.

Let us assume that we have reduced photometric observations of $N$ mutual events. For each event we then choose an instant of time as is described above and thereby obtain a series of such instants, $t^{(1)}, t^{(2)}, \ldots, t^{(N)}$ and the corresponding series of parameters $X_{k}^{(\mathrm{o})}, Y_{k}^{(\mathrm{o})} \quad(k=$ $1,2, \ldots, N)$, determined by relations (3). These parameters play the part of measured values of $X_{\mathrm{a}}, Y_{\mathrm{a}}$.

We further assume that before carrying out the second stage we have at our disposal an approximate theory of satellite motion and approximate elements of satellite orbits inferred from earlier observations. It may be another theory, which differs from that used to reduce photometric observations. The formulas of this theory and relations (1), (2) can be used to compute $X_{\mathrm{a}}, Y_{\mathrm{a}}$, which correspond to observing times $t^{(1)}, t^{(2)}, \ldots, t^{(N)}$. We denote this series of quantities as $X_{k}^{(\mathrm{c})}, Y_{k}^{(\mathrm{c})}(k=1,2, \ldots, N)$. These quantities play the part of computed theoretical values of $X_{\mathrm{a}}$, $Y_{\mathrm{a}}$.

We now denote the orbital elements of the passive satellite as $p_{1}, p_{2}, \ldots, p_{n}$. Depending on the theory used, they can be different from the elements of the Keplerian orbit and the number $n$ of these parameters can exceed six. We denote the corresponding orbital elements of the active satellite as $q_{1}, q_{2}, \ldots, q_{n}$.

We can now write the conditional equations which are used to refine the elements of satellite orbits. According to the general formulation of the method of differential adjusting and in view of relations (1), (2) the conditional equations can be written in the following form:

$X_{k}^{(\mathrm{o})}-X_{k}^{(\mathrm{c})}$

$$
\begin{aligned}
& =\sum_{j=1}^{n}\left[a_{x}\left(\frac{\partial x_{\mathrm{a}}}{\partial q_{j}}\right)_{0}+a_{y}\left(\frac{\partial y_{\mathrm{a}}}{\partial q_{j}}\right)_{0}+a_{z}\left(\frac{\partial z_{\mathrm{a}}}{\partial q_{j}}\right)_{0}\right] \Delta q_{j} \\
& -\sum_{j=1}^{n}\left[a_{x}\left(\frac{\partial x_{\mathrm{p}}}{\partial p_{j}}\right)_{0}+a_{y}\left(\frac{\partial y_{\mathrm{p}}}{\partial p_{j}}\right)_{0}+a_{z}\left(\frac{\partial z_{\mathrm{p}}}{\partial p_{j}}\right)_{0}\right] \Delta p_{j} \\
& Y_{k}^{(\mathrm{o})}-Y_{k}^{(\mathrm{c})} \\
& =\sum_{j=1}^{n}\left[b_{x}\left(\frac{\partial x_{\mathrm{a}}}{\partial q_{j}}\right)_{0}+b_{y}\left(\frac{\partial y_{\mathrm{a}}}{\partial q_{j}}\right)_{0}+b_{z}\left(\frac{\partial z_{\mathrm{a}}}{\partial q_{j}}\right)_{0}\right] \Delta q_{j} \\
& -\sum_{j=1}^{n}\left[b_{x}\left(\frac{\partial x_{\mathrm{p}}}{\partial p_{j}}\right)_{0}+b_{y}\left(\frac{\partial y_{\mathrm{p}}}{\partial p_{j}}\right)_{0}+b_{z}\left(\frac{\partial z_{\mathrm{p}}}{\partial p_{j}}\right)_{0}\right] \Delta p_{j}
\end{aligned}
$$$$
(k=1,2, \ldots, N) \text {. }
$$

where $\Delta p_{j}, \Delta q_{j}$ are the corrections to be found for the preliminary values of the elements.

Here partial derivatives of $x_{\mathrm{a}}, y_{\mathrm{a}}, z_{\mathrm{a}}$ are computed at time $t_{\mathrm{a}}$, and partial derivatives of $x_{\mathrm{p}}, y_{\mathrm{p}}, z_{\mathrm{p}}$, at time $t_{\mathrm{p}}$ corresponding to time $t^{(k)}$. To compute these derivatives, we use the formulae of the theory of motion of satellites and preliminary orbital elements. The necessary values of $t_{\mathrm{a}}, t_{\mathrm{p}}, a_{x}, a_{y}, a_{z}, b_{x}, b_{y}, b_{z}$ are known and saved from the observation reduction.

The conditional equations thus constructed can be used, if combined with other conditional equations inferred from other types of observations with convenient weights, to refine the elements of satellite orbits.

\section{Results of the reduction of photometric observations}

\subsection{The notation}

We recorded observing times on the $U T C$ scale and converted them into the $T T$ scale prior to reduction. To this end, we used the following relation:

$T T=U T C+63.184$ s.

The results of the reduction of photometric observations are given in Tables 3 and 4, and in Figs. 1-31. We describe now the notation that we used to present the results of this paper.

$t_{0}$ is the time of observation, MJD (MJD $=\mathrm{JD}-$ $2400000.5)$ with fractions in the $T T$ time scale. This quantity is not necessary for subsequent determination of the elements of the satellite orbit, however, it can be used to control and identify the data. For each observed event the time $t_{0}$ was chosen arbitrary within the period of phenomenon.

$t_{\mathrm{a}}, t_{\mathrm{p}}$ are the times as explained above, MJD with fractions in the $T T$ time scale.

$X^{(\mathrm{o})}, Y^{(\mathrm{o})}$ are two components (in kilometers) of the mutual position vector of satellites calculated using formulas (3) as the main results of the reduction of photometric observations. 
$\sigma X, \sigma Y$ are the standard errors of $X^{(\mathrm{o})}, Y^{(\mathrm{o})}$ as inferred from the reduction of photometric observations (in kilometers). These errors can be used in subsequent computations to determine the weights in the least squares method.

$a_{x}, a_{y}, a_{z}, b_{x}, b_{y}, b_{z}$ : the dimensionless coefficient required for application of the results obtained (see formula $(2)$ ). The value of $a_{z}$ is always equal to zero.

The results presented also include corrections $D_{x}, D_{y}$, which incorporate the contributions due to observational errors and the errors of the theory. These corrections characterize the agreement between theory and observations $(\mathrm{O}-\mathrm{C})$.

The accuracy of the relative satellite positions inferred from photometric observations of mutual events is characterized by standard errors $\sigma X, \sigma Y$ expressed as linear quantities. It is interesting to compare these errors with the accuracy of ground-based angle measurements expressed in arcseconds. The observational errors of the two different types can be co-ordinated by taking into account the angles at which intervals $X_{\mathrm{a}}$ and $Y_{\mathrm{a}}-$ the projections of the satellite - satellite vector on the sky plane located at a topocentric distance to the active satellite are seen from the Earth. This is the case when mutual occultations of satellites are considered. When analyzing the mutual eclipses the sky plane is perpendicular to the heliocentric direction of the eclipsed satellite. In this case the angles corresponding to intervals $X_{\mathrm{a}}$ and $Y_{\mathrm{a}}$ are heliocentric angles. The accuracy of the determination of these angles is then similar to that of ground-based photographic angle measurements. Therefore along the standard errors $\sigma X, \sigma Y$ we also give the corresponding angular errors $\sigma_{\mathrm{a}} X, \sigma_{\mathrm{a}} Y$ computed using the following formulas:

$\sigma_{\mathrm{a}} X=\operatorname{arctg}\left(\frac{\sigma X}{r}\right), \quad \sigma_{\mathrm{a}} Y=\operatorname{arctg}\left(\frac{\sigma Y}{r}\right)$,

where $r$ is the topocentric or heliocentric distance of the active satellite depending on the type of the observed event. Standard errors $\sigma_{\mathrm{a}} X, \sigma_{\mathrm{a}} Y$ given in Table 4 are in arcsec.

We number the Galilean satellites in accordance with the generally accepted numbering system: Io - 1, Europa2, Ganymede -3 , and Callisto - 4 .

We identify mutual phenomena of the Galilean satellites by assigning to them the codes consisting of date (yymmdd) and the following values separated by dash: $n_{\mathrm{a}}$ (number of active satellite), $P$ - type of mutual phenomenon, $n_{\mathrm{p}}$ (number of passive satellite). $P$ is $\mathrm{O}$ for $\mathrm{mu}-$ tual occultation and $P$ is $\mathrm{E}$ for mutual eclipse. For example, identifier 970803-4E1 refers to Io's eclipse by Callisto on August 3, 1997.

Each event could have been observed at several observatories yielding several light curves. To designate the particular light curve, we add the observer's code to the event identifier (see Table 1). This conventional code identifies the observatory, observers, and the equipment involved in each particular observation. Thus, if phenomenon 9708034E1 was observed at the Pulkovo observatory, the results should be coded as 970803-4E1-k. The results of observations of the same phenomenon made at the Nauchny observatory by observer Irsmambetova T.R. using photoelectric photometer will be referred to via identifier 970803$4 \mathrm{E} 1-\mathrm{t}$.

\subsection{Description of the results}

Each line in Tables 3 and 4 corresponds to observation of one event by one observer, i.e., refers to one light curve.

Table 3 contains the data required to refine the elements of satellite orbits. Table 4 gives the parameters that allow the accuracy and reliability of results to be assessed. To establish a correspondence between the lines in two tables that refer to the results of the same observation, we give the observation identifier in the first column of each table.

The results of reduction of observations $X^{(\mathrm{o})}$ and $Y^{(\mathrm{o})}$ depend substantially on time $t_{0}$, which was set equal to one of the observing times near the light minimum of the passive satellite. The sets of observing times for the same event differ from one observatory to another. Therefore $X^{(\mathrm{o})}$ and $Y^{(\mathrm{o})}$ inferred from observations made at different observatories cannot be compared to each other. By contrast, we assume $D_{x}$ and $D_{y}$ to be constant throughout the particular phenomenon as is explained above allowing us to compare $D_{x}$ and $D_{y}$ values inferred from observations performed at different observatories. The discrepancies between values inferred from observations made at different observatories are therefore due to observational errors, thereby providing an external estimate for the latter. Parameters $\sigma X$ and $\sigma Y$ characterize the internal observational errors.

The accuracy of the photometric observations performed and the quality of the data obtained can be assessed from graphs illustrating the agreement between the theory and observations (Figs. 1-31). The dots or other symbols show the satellite flux, $S_{i}$, corresponding to the measured photometric count $E_{i}$ and parameters $D_{x}, D_{y}, K, Q$, and $L, P$ obtained from the process of the reduction of observations using formula

$S_{i}=\frac{E_{i}-P-L\left(t_{i}-t_{\mathrm{b}}\right)}{K+Q\left(t_{i}-t_{\mathrm{b}}\right)}$

The model of mutual occultation or eclipse of the satellites is used to compute and plot theoretical satellite fluxes, $S\left(X_{\mathrm{t}}+D_{x}, Y_{\mathrm{t}}+D_{y}\right)$, for each measurement time. Theoretical values on graphs are connected by a continuous curve. Each graph (the curve and the dots) represents the results of a single observation of one event at one observatory and is identified as explained above, e.g., 9708034E1-t in the case of Io's eclipse by Callisto on August 3, 1997 observed by Irsmambetova T.R. at the Nauchny observatory. We plot in the same figure the results of all 
Table 3. Relative positions of the Galilean satellites derived from the photometric observations

\begin{tabular}{|c|c|c|c|c|c|c|c|c|c|}
\hline Observation & $t_{\mathrm{a}}$ & $t_{\mathrm{p}}$ & $X^{(\mathrm{o})}$ & $Y^{(\mathrm{o})}$ & $a_{x}$ & $a_{y}$ & $b_{x}$ & $b_{y}$ & $\overline{b_{z}}$ \\
\hline 970413-1O2-d & 50550.990029 & 50550.989991 & 379 & -964 & 0.647097 & 0.762407 & 0.213239 & -0.180988 & 0.960090 \\
\hline 970422-4O3-d & 50560.963826 & 50560.963768 & -218 & 540 & 0.626288 & 0.779592 & 0.212176 & -0.170452 & 0.962251 \\
\hline 970622-1E2-a & 50621.854428 & 50621.854459 & 531 & -1396 & 0.700797 & 0.713360 & 0.216057 & -0.212252 & 0.953031 \\
\hline 970706-3E4-v & 50635.913714 & 50635.913775 & -674 & 2058 & 0.685900 & 0.727696 & 0.216626 & -0.204184 & 0.954663 \\
\hline 970715-1O3-a & 50644.767217 & 50644.767163 & 1020 & -2570 & 0.605868 & 0.795565 & 0.214879 & -0.163643 & 0.962833 \\
\hline 970718-3E2-a & 50647.766400 & 50647.766442 & -1108 & 3155 & 0.670902 & 0.741545 & 0.216719 & -0.196073 & 0.956341 \\
\hline 970718-3O2-a & 50647.843641 & 50647.843601 & 491 & -1443 & 0.609167 & 0.793042 & 0.215321 & -0.165397 & 0.962435 \\
\hline 970718-3O2-g & 50647.843647 & 50647.843607 & 531 & -1551 & 0.609165 & 0.793043 & 0.215322 & -0.165397 & 0.962434 \\
\hline 970719-3O1-a & 50648.851532 & 50648.851479 & 400 & -1055 & 0.611784 & 0.791025 & 0.215666 & -0.166798 & 0.962116 \\
\hline 970724-1E4-a & 50653.741856 & 50653.741934 & 818 & -2368 & 0.665072 & 0.746779 & 0.216751 & -0.193036 & 0.956951 \\
\hline 970725-1E4-a & 50654.846134 & 50654.846210 & -676 & 1823 & 0.663051 & 0.748574 & 0.216693 & -0.191937 & 0.957186 \\
\hline 970725-1E4-t & 50654.846106 & 50654.846182 & -533 & 1678 & 0.663051 & 0.748574 & 0.216693 & -0.191936 & 0.957186 \\
\hline 970725-1E4-g & 50654.846302 & 50654.846378 & -649 & 1880 & 0.663051 & 0.748574 & 0.216693 & -0.191936 & 0.957186 \\
\hline 970725-3E2-a & 50654.916027 & 50654.916066 & -1037 & 2996 & 0.662727 & 0.748861 & 0.216675 & -0.191753 & 0.957227 \\
\hline 970725-3E2-k & 50654.915886 & 50654.915926 & -1021 & 2998 & 0.662727 & 0.748861 & 0.216675 & -0.191753 & 0.957227 \\
\hline 970725-3E2-g & 50654.915801 & 50654.915841 & -997 & 2991 & 0.662727 & 0.748861 & 0.216675 & -0.191753 & 0.957227 \\
\hline $970725-3 \mathrm{O} 2-\mathrm{k}$ & 50654.969840 & 50654.969801 & 556 & -1432 & 0.620168 & 0.784469 & 0.216654 & -0.171277 & 0.961106 \\
\hline $970725-3 \mathrm{O} 2-\mathrm{t}$ & 50654.970338 & 50654.97 & 515 & -1521 & 0.620169 & 0.784468 & 0.216653 & -0.171277 & 106 \\
\hline 970731-4E3-e & 50660.991690 & 50660.991752 & 414 & -1170 & 0.655323 & 0.755349 & 0.216531 & -0.187857 & 0.958031 \\
\hline 970801-4E2-g & 50661.818732 & 50661.818817 & 1076 & -3235 & 0.654879 & 0.755734 & 0.216545 & -0.187647 & 0.958069 \\
\hline 970801-4E2-1 & 50661.818615 & 50661.818700 & 1012 & -2951 & 0.654879 & 0.755733 & 0.216545 & -0.187647 & 0.958069 \\
\hline $970801-4 \mathrm{E} 3-\mathrm{k}$ & 50660.991652 & 50660.991714 & 423 & -1195 & 323 & 0.75 & 0.21 & -0.1 & 031 \\
\hline $970803-4 \mathrm{E} 1-\mathrm{k}$ & 50662.980645 & 50662.980730 & -325 & 773 & 0.654328 & 0.756211 & 0.216578 & -0.187399 & 0.958110 \\
\hline 970803-4E1-1 & 50662.980643 & 50662.980728 & -134 & 372 & 0.654328 & 0.756211 & 0.216578 & -0.187399 & 0.958110 \\
\hline $970803-4 \mathrm{E} 1-\mathrm{t}$ & 50662.980706 & 50662.980791 & -311 & 886 & 0.654328 & 0.756211 & 0.216578 & -0.187399 & 0.958110 \\
\hline 970830-3E2-a & 50690.754789 & 50690.754816 & -1214 & 3592 & 0.620757 & 0.784003 & 0.214981 & -0.170217 & 0.961670 \\
\hline 970830-3O2-a & 50690.646325 & 50690.646296 & -614 & 1892 & 0.678793 & 0.734330 & 0.220080 & -0.203436 & 0.954033 \\
\hline 970903-1E3-a & 50694.620004 & 50694.620040 & -79 & 194 & 0.617212 & 0.786797 & 0.214789 & -0.168494 & 0.962016 \\
\hline 970906-3O2-g & 50697.809825 & 50697.809798 & -746 & 2432 & 0.688229 & 0.725493 & 0.220037 & -0.208735 & 0.952897 \\
\hline 970910-1O3-a & 50701.655382 & 50701.655345 & -222 & 646 & 0.693845 & 0.720125 & 0.219966 & -0.211938 & 0.952206 \\
\hline 970914-3E1-e & 50705.716657 & 50705.716714 & 323 & -526 & 0.603294 & 0.797519 & 0.213624 & -0.161598 & 0.963458 \\
\hline 970915-3E2-a & 50706.608493 & 50706.608526 & -173 & -445 & 0.603013 & 0.797731 & 0.213642 & -0.161495 & 0.963471 \\
\hline 970918-1O3-g & 50709.687811 & 50709.687766 & -967 & 2883 & 0.700427 & 0.713724 & 0.219700 & -0.215607 & 0.951444 \\
\hline 970918-1E3-e & 50709.791892 & 50709.791940 & -166 & 467 & 0.598091 & 0.801428 & 0.213127 & -0.159053 & 0.963991 \\
\hline 970918-1E3-t & 50709.791845 & 50709.791893 & -138 & 526 & 0.598091 & 0.801428 & 0.213127 & -0.159053 & 0.963991 \\
\hline 970921-3E1-1 & 50712.831184 & 50712.831240 & 462 & -1201 & 0.594581 & 0.804035 & 0.212791 & -0.157358 & 0.964343 \\
\hline 970922-3E2-g & 50713.768904 & 50713.768940 & 928 & -2302 & 0.594290 & 0.804251 & 0.212808 & -0.157252 & 0.964357 \\
\hline 971007-4O1-t & 50728.790911 & 50728.790826 & 1185 & -3373 & 0.708427 & 0.705784 & 0.219125 & -0.219946 & 0.950583 \\
\hline 971109-4O2-t & 50761.672069 & 50761.672005 & 1390 & -2436 & 0.686812 & 0.726835 & 0.219121 & -0.207056 & 0.953474 \\
\hline 971109-4O2-w & 50761.672881 & 50761.672816 & 918 & -2967 & 0.686812 & 0.726835 & 0.219122 & -0.207056 & 0.953474 \\
\hline 971110-3E1-t & 50762.710973 & 50762.711012 & 1139 & -2885 & 0.531928 & 0.846789 & 0.204298 & -0.128334 & 0.970460 \\
\hline 971118-3O1-g & 50770.562083 & 50770.562049 & -1155 & 3335 & 0.674907 & 0.737903 & 0.219004 & -0.200308 & 0.954942 \\
\hline
\end{tabular}

observations of the same event made simultaneously at several observatories. Therefore some of the figures show multiple satellite light curves thereby allowing the results obtained at different observatories to be compared and the systematic errors made during observations to be revealed.

Some light curves were obtained with CCD camera. In this case zero value of satellite flux $S$ corresponds to zero value of $E$. This make it possible to put $P=0, L=0$. No observation allowed us to determine all the parameters $K, Q, P, L$ together empirically. The more detailed comments to each light curve reduced are in the following subsection.

For each observation Table 4 contains the reference (Fig) to the corresponding figure, the reference $(\mathrm{C})$ to the special comment, and the list $(E(S))$ of the parameters from the set $K, Q, P, L$ which were really determined. To each observation we assigned a quality index $Q$. The value $P$ (perfect) of the quality index means that the list of the parameters determined fully corresponds to the method of observation. The value $V$ (vague) shows that the only parameter $K$ could be determined and that real error of the values $X^{(\mathrm{o})}, Y^{(\mathrm{o})}$ may be more important than the estimations $\sigma X, \sigma Y$.

\subsection{Comments and remarks on the observations}

Each of following comments is attached to some light curve according to the reference $\mathrm{C}$ in Table 4.

1. Measurements of the flux from occulted and occulting satellites or the flux from eclipsed satellites relative to the flux from the reference satellite were made by the CCD detector so the variations of the air mass transparency were taken into account. In this case the parameter $Q$ may be put at zero or determined from observations;

2. Measurements of the flux from the occulted and occulting satellites or from the eclipsed satellite were made 
Table 4. The fit of the theory to the photometric observations and precision estimates

\begin{tabular}{|c|c|c|c|c|c|c|c|c|c|c|c|}
\hline Observation & \begin{tabular}{l|} 
Fig | \\
\end{tabular} & $t_{0}$ & $D_{x}, \mathrm{~km}$ & $D_{y}, \mathrm{~km}$ & $\sigma X, \mathrm{~km}$ & $\sigma Y, \mathrm{~km}$ & $\sigma_{\mathrm{a}} X,,^{\prime \prime}$ & $\sigma_{\mathrm{a}} Y^{\prime \prime}$ & $E(S)$ & $\mathrm{Q}$ & $\mathrm{C}$ \\
\hline $970413-1 \mathrm{O} 2-\mathrm{d}$ & 1 & 50551.021346 & 571 & 56 & 24 & 27 & 0.006 & 0.007 & $\mathrm{~K}$ & $\mathrm{~V}$ & 6 \\
\hline $970422-4 \mathrm{O} 3-\mathrm{d}$ & 2 & 50560.994237 & 299 & -573 & 34 & 78 & 0.009 & 0.020 & KPL & $\mathrm{P}$ & 4 \\
\hline 970622-1E2-a & 3 & 50621.879701 & 291 & 222 & 44 & 32 & 0.012 & 0.008 & KQ & $\mathrm{P}$ & 1 \\
\hline 970706-3E4-v & 4 & 50635.938173 & -959 & -156 & 107 & 99 & 0.029 & 0.027 & $\mathrm{~K}$ & $\mathrm{~V}$ & 7 \\
\hline 970715-1O3-а & 5 & 50644.791125 & 218 & -419 & 63 & 42 & 0.021 & 0.014 & KQ & $\mathrm{P}$ & 2 \\
\hline 970718-3E2-а & 6 & 50647.790257 & 104 & -15 & 93 & 46 & 0.025 & 0.012 & $\mathrm{KQ}$ & $\mathrm{P}$ & 1 \\
\hline 970718-3O2-a & 7 & 50647.867410 & -74 & 327 & 7 & 7 & 0.002 & 0.002 & $\mathrm{KQ}$ & $\mathrm{P}$ & 2 \\
\hline $970718-3 \mathrm{O} 2-\mathrm{g}$ & 7 & 50647.867415 & -31 & 223 & 12 & 13 & 0.004 & 0.004 & $\mathrm{~K}$ & $\mathrm{~V}$ & 6 \\
\hline 970719-3O1-а & 8 & 50648.875257 & -80 & 88 & 19 & 23 & 0.006 & 0.008 & $\mathrm{KQ}$ & $\mathrm{P}$ & 2 \\
\hline 970724-1E4-a & 9 & 50653.765616 & 944 & -2155 & 165 & 106 & 0.045 & 0.029 & $\mathrm{KQ}$ & $\mathrm{P}$ & 1 \\
\hline 970725-1E4-а & 10 & 50654.869863 & -118 & -175 & 73 & 196 & 0.020 & 0.054 & $\mathrm{~K}$ & $\mathrm{~V}$ & 5 \\
\hline 970725-1E4-t & 10 & 50654.869835 & -1 & -330 & 28 & 27 & 0.007 & 0.007 & $\mathrm{~K}$ & $\mathrm{~V}$ & 6 \\
\hline 970725-1E4-g & 10 & 50654.870031 & 91 & -54 & 31 & 28 & 0.008 & 0.008 & $\mathrm{~K}$ & $\mathrm{~V}$ & 6 \\
\hline 970725-3Е2-a & 11 & 50654.939655 & 142 & 116 & 96 & 51 & 0.026 & 0.014 & KQ & $\mathrm{P}$ & 1 \\
\hline $970725-3 \mathrm{E} 2-\mathrm{k}$ & 11 & 50654.939515 & 20 & 71 & 105 & 58 & 0.029 & 0.016 & $\mathrm{~K}$ & $\mathrm{P}$ & 1 \\
\hline $970725-3 \mathrm{E} 2-\mathrm{g}$ & 11 & 50654.939429 & -41 & 36 & 71 & 36 & 0.019 & 0.010 & $\mathrm{~K}$ & $\mathrm{~V}$ & 6 \\
\hline $970725-3 \mathrm{O} 2-\mathrm{k}$ & 12 & 50654.993386 & -341 & -255 & 77 & 96 & 0.026 & 0.032 & K & $\mathrm{V}$ & 5 \\
\hline $970725-3 \mathrm{O} 2-\mathrm{t}$ & 12 & 50654.993885 & 87 & -179 & 6 & 16 & 0.002 & 0.006 & KPL & $\mathrm{P}$ & 4 \\
\hline 970731-4E3-e & 13 & 50661.015210 & 152 & 112 & 12 & 15 & 0.003 & 0.004 & KQ & $\mathrm{P}$ & 2 \\
\hline $970801-4 \mathrm{E} 2-\mathrm{g}$ & 14 & 50661.842276 & 371 & 101 & 151 & 92 & 0.041 & 0.025 & $\mathrm{~K}$ & $\mathrm{~V}$ & 6 \\
\hline 970801-4E2-1 & 14 & 50661.842159 & 157 & 333 & 57 & 39 & 0.015 & 0.011 & $\mathrm{~K}$ & $\mathrm{~V}$ & 6 \\
\hline 970801-4E3-k & 15 & 50661.015172 & 138 & 80 & 52 & 55 & 0.014 & 0.015 & $\mathrm{KQ}$ & $\mathrm{P}$ & 1 \\
\hline 970803-4E1-k & 16 & 50663.004171 & 55 & 302 & 4 & 6 & 0.001 & 0.002 & $\mathrm{KQ}$ & $\mathrm{P}$ & 1 \\
\hline 970803-4E1-1 & 16 & 50663.004169 & 242 & -101 & 31 & 70 & 0.008 & 0.019 & $\mathrm{~K}$ & $\mathrm{~V}$ & 6 \\
\hline 970803-4E1-t & 16 & 50663.004232 & 172 & 426 & 163 & 451 & 0.044 & 0.123 & KPL & $\mathrm{P}$ & 4 \\
\hline 970830-3Е2-а & 17 & 50690.778555 & -1517 & -495 & 138 & 60 & 0.038 & 0.016 & $\mathrm{KQ}$ & $\mathrm{P}$ & 1 \\
\hline 970830-3O2-a & 18 & 50690.670037 & 128 & -47 & 7 & 5 & 0.002 & 0.002 & KQ & $\mathrm{P}$ & 2 \\
\hline 970903-1E3-a & 19 & 50694.643972 & 128 & -402 & 53 & 141 & 0.014 & 0.038 & $\mathrm{KQ}$ & $\mathrm{P}$ & 1 \\
\hline 970906-3O2-g & 20 & 50697.833822 & 272 & -454 & 24 & 18 & 0.008 & 0.006 & $\mathrm{~K}$ & $\mathrm{~V}$ & 6 \\
\hline 970910-1O3-а & 21 & 50701.679597 & 65 & -81 & 16 & 27 & 0.005 & 0.009 & $\mathrm{KQ}$ & $\mathrm{P}$ & 2 \\
\hline 9709 & 22 & 50705.741160 & -88 & 18 & 13 & 24 & 0.004 & 0.006 & $\mathrm{KQ}$ & $\mathrm{P}$ & 2 \\
\hline$-3 \mathrm{E} 2-\mathrm{a}$ & 23 & 50706.633011 & -54 & 569 & 71 & 160 & 0.019 & 0.044 & $\mathrm{KQ}$ & $\mathrm{P}$ & 1 \\
\hline 970918-1O3-g & 24 & 50709.712467 & 160 & 75 & 63 & 39 & 0.020 & 0.012 & $\mathrm{~K}$ & $\mathrm{~V}$ & 6 \\
\hline$-1 \mathrm{E} 3-\mathrm{e}$ & 25 & 50709.816646 & -23 & 267 & 11 & 21 & 0.003 & 0.006 & $\mathrm{KQ}$ & $\mathrm{P}$ & 2 \\
\hline$-1 \mathrm{E} 3-\mathrm{t}$ & 25 & 50709.816599 & -71 & 300 & 22 & 46 & 0.006 & 0.013 & $\mathrm{~K}$ & $\mathrm{~V}$ & 6 \\
\hline 970921-3E1-1 & 26 & 50712.856114 & 821 & 598 & 16 & 19 & 0.004 & 0.005 & $\mathrm{~K}$ & $\mathrm{~V}$ & 6 \\
\hline 970922-3E2-g & 27 & 50713.793868 & 189 & -282 & 49 & 106 & 0.014 & 0.029 & KPL & $\mathrm{P}$ & 3 \\
\hline 971007-4O1-t & 28 & 50728.816855 & -248 & -37 & 37 & 43 & 0.011 & 0.013 & KPL & $\mathrm{P}$ & 4 \\
\hline $971109-4 \mathrm{O} 2-\mathrm{t}$ & 29 & 50761.700869 & 516 & -373 & 10 & 7 & 0.003 & 0.002 & $\mathrm{~K}$ & $\mathrm{~V}$ & 6 \\
\hline 9711 & 29 & 50761.7 & 397 & -796 & 31 & 47 & 0.009 & 0.013 & KPL & $\mathrm{P}$ & 8 \\
\hline 971110-3E1-t & 30 & 50762.739970 & 1425 & 2503 & 262 & 555 & 0.072 & 0.152 & KPL & $\mathrm{P}$ & 4 \\
\hline 971118-3O1-g & 31 & 50770.591704 & 245 & 248 & 26 & 16 & 0.007 & 0.004 & $\mathrm{~K}$ & $\mathrm{~V}$ & 6 \\
\hline
\end{tabular}

by the CCD detector. The parameters $K, Q$ were successfully determined as necessary;

3. Measurements of the flux from occulted or eclipsed satellite relative to the flux from the reference satellite were made by the photometer so the variations of the air mass transparency were taken into account. The parameters $K, P, L$ were successfully determined as necessary;

4. Measurements of the direct flux from the occulted and occulting satellites or from the eclipsed satellite were made through the diaphragm by the photometer and four parameters $K, Q, P, L$ are to be determined. However the data allowed the only parameters $K, P, L$ to be successfully determined so the variations of the air mass transparency were only partially taken into account;

5. Measurements of the flux from the occulted and occulting satellites or from the eclipsed satellite were made by the CCD receiver. The parameters $K, Q$ are to be determined. However the data do not allow us to determine the parameter $Q$ and only parameter $K$ was determined. The real errors may be more important than the estimates $\sigma X$ and $\sigma Y$;

6. Measurements of the flux by the photometer were made. The data do not allow us to determine the parameters $Q, P, L$ and only parameter $K$ was determined. The real errors may be more important than the estimates $\sigma X$ and $\sigma Y$;

7. Visual measurements of the flux from the eclipsed satellite were made and the parameters $K, P, L$ were determined;

8. Visual measurements of the flux from the occulted and occulting satellites were made and only parameter $K$ was determined. The real errors may be more important than the estimates $\sigma X$ and $\sigma Y$. 


\section{Conclusions}

The results reported in this paper can be used directly to refine the orbital elements of the Galilean satellites. It should be kept in mind that a refined set of satellite orbital elements can be inferred from the entire data base of available observations of these satellites. The new portion of observational data obtained as a result of the observational campaign performed contributes significantly to the overall data base of the events considered.

The estimates made in this paper show that the satellite positional data inferred from photometric observations of mutual occultations and eclipses of the satellites considered proves to be several tens to several hundreds of times more accurate than the results of the best groundbased photographic observations. We obtained our data with rather modest observational equipment available at the observatories that took part in the campaign.

A preliminary analysis of the accuracy estimates reported in this paper and inspection of plots illustrating the observational results led us to the following conclusions. In some cases the discrepancies between the results of observations of the same event at different observatories exceed the estimated internal errors of each observation, which is indicative of certain systematic errors.

The greatest error in positional data inferred from such external estimates is due to errors of photometric measurements. Only in some specific cases have there been significant discrepancies between observing times. This leads us to a preliminary conclusion that such observations require more accurate allowance for interfering factors. The latter can include scattered light falling into photometer or distortions introduced by the detector. A more detailed analysis of observations is needed.
We can finally conclude that the observing campaign carried out by observatories in Kazakhstan, Russia, and Ukraine in 1997 yielded the expected result. The number of successful attempts increased substantially compared to previous similar observations carried out in 1995 and earlier. To improve this very efficient technique for extracting astrometric information about planetary satellites, the observatories need to be provided with new observing equipment.

Acknowledgements. This work was supported by the Russian Foundation for Basic Research, project No. 97-02-16551.

\section{References}

Aksnes K., Franklin F., Millis R., Birch P., Blanco C., Catalano S., 1984, AJ 89, 280

Arlot J.-E., 1984, A\&A 138, 113

Arlot J.-E., Thuillot W., Barroso J. Jr., et al., 1992, A\&AS 92, 151

Grigorjeva Z.N., Egorov Yu.A., Tejfel V.G., Charitonova G.A., 1986a, Astron. Circ. 1444, 1

Grigorjeva Z.N., Egorov Yu.A., Tejfel V.G., Charitonova G.A., 1986b, Astron. Circ. 1447, 3

Emel'yanov N.V., 1995, AZh 71, 794

Emel'yanov N.V., Irsmambetova T.R., Tejfel V.G., 1997, Astron. Vestnik 31, 491

Emel'yanov N.V., 1999, Astron. Vestnik 33, 154

Emel'yanov N.V., 2000, Astron. Vestnik 34 (in press)

Nasonova L.P., 1996, Lett. AZh 22, 157

Thuillot W., Morando B., Modélisation de courbes photométriques de phénomènes mutuels des satellites galiléens de Jupiter, Notes scientifiques et techniques du Bureau des Longitudes, S030 


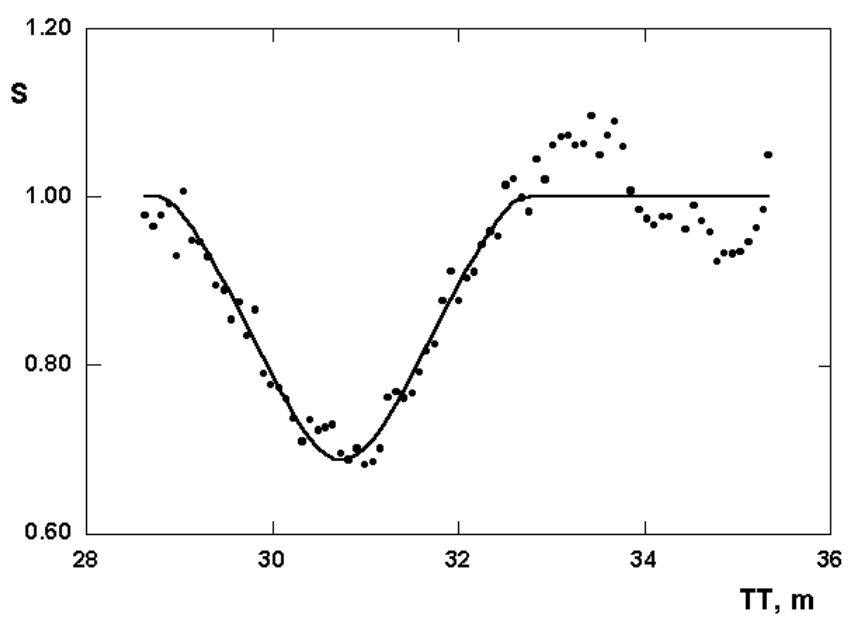

Fig. 1. The fit of the theory to the photometric observations 970413-1O2-d

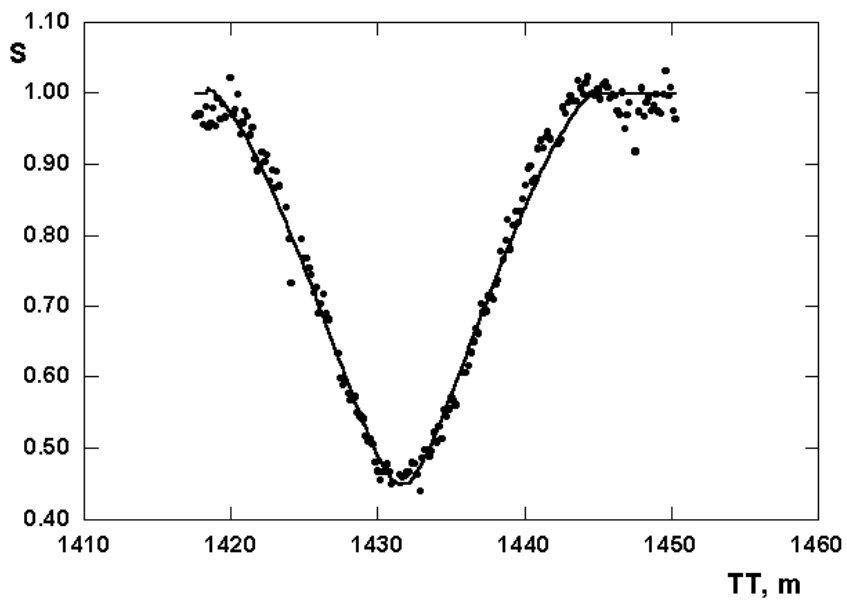

Fig. 2. The fit of the theory to the photometric observations 970422-4O3-d. The time is measured from $0^{\mathrm{h}}$ Apr. 22, 1997

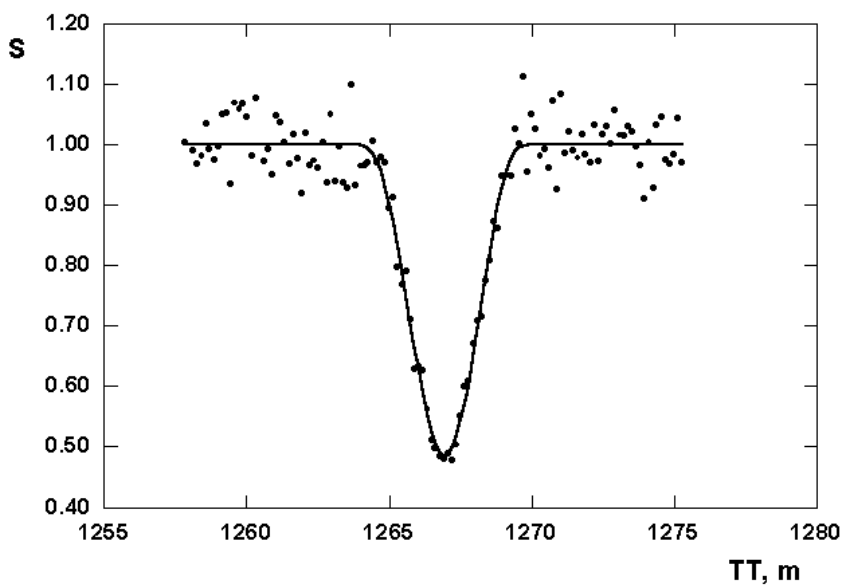

Fig. 3. The fit of the theory to the photometric observations 970622-1E2-a

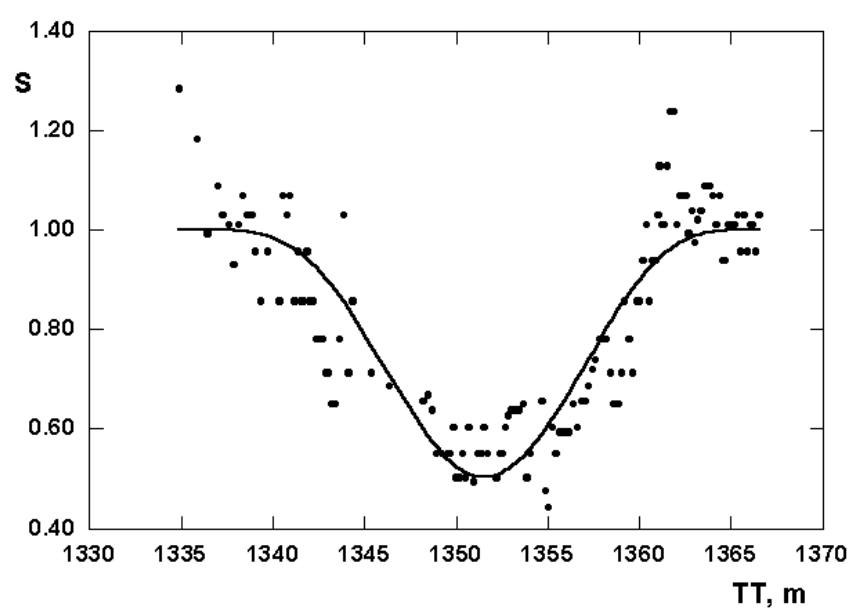

Fig. 4. The fit of the theory to the photometric observations 970706-3E4-v

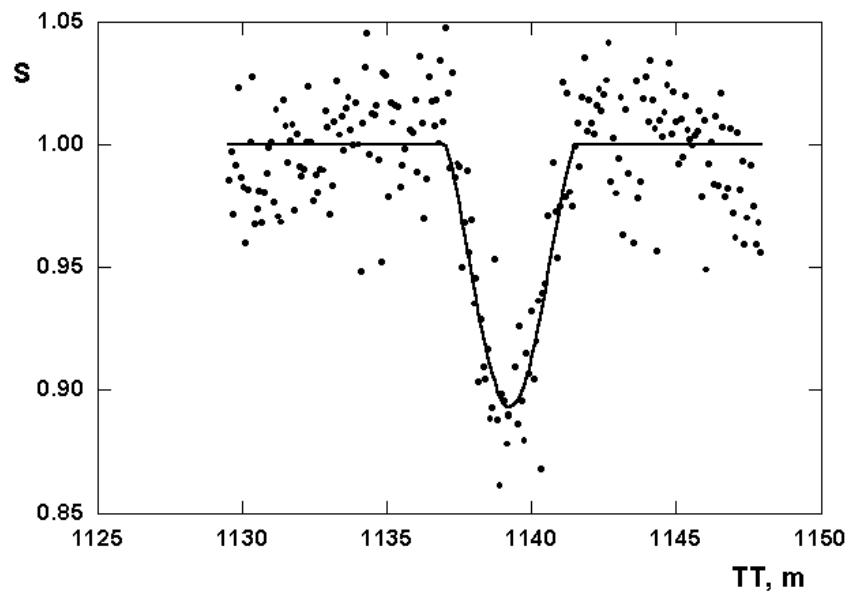

Fig. 5. The fit of the theory to the photometric observations 970715-1O3-a

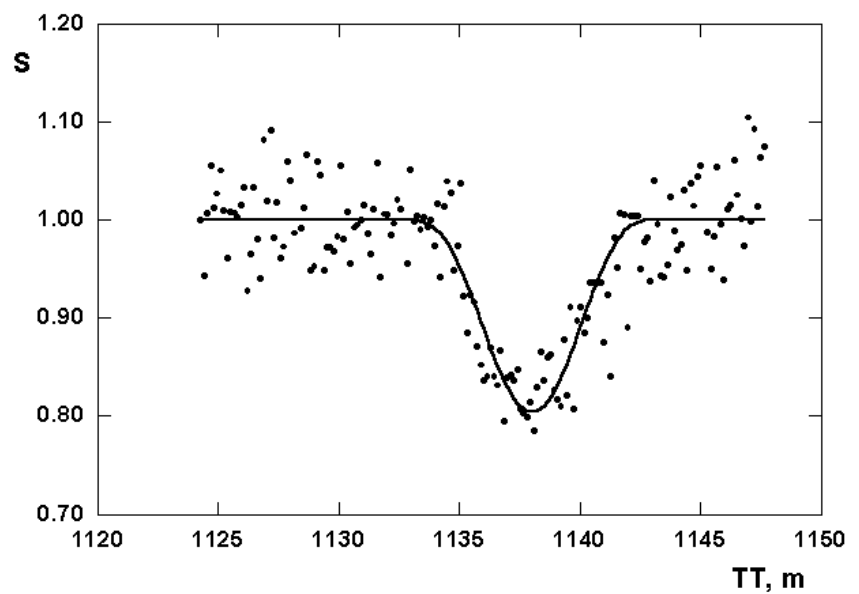

Fig. 6. The fit of the theory to the photometric observations 970718-3E2-a 


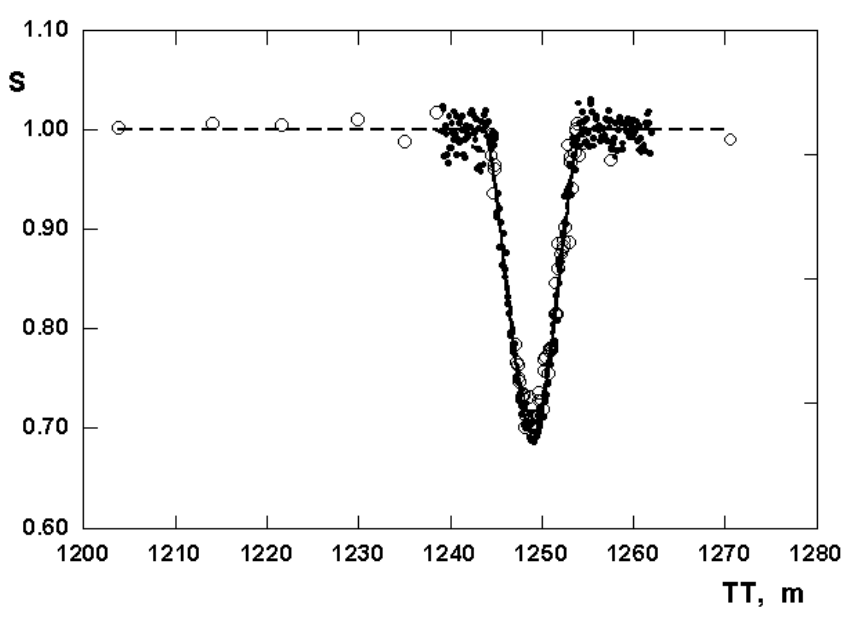

Fig. 7. The fit of the theory to the photometric observations 970718-3O2-a (dots) and 970718-3O2-g (circles)

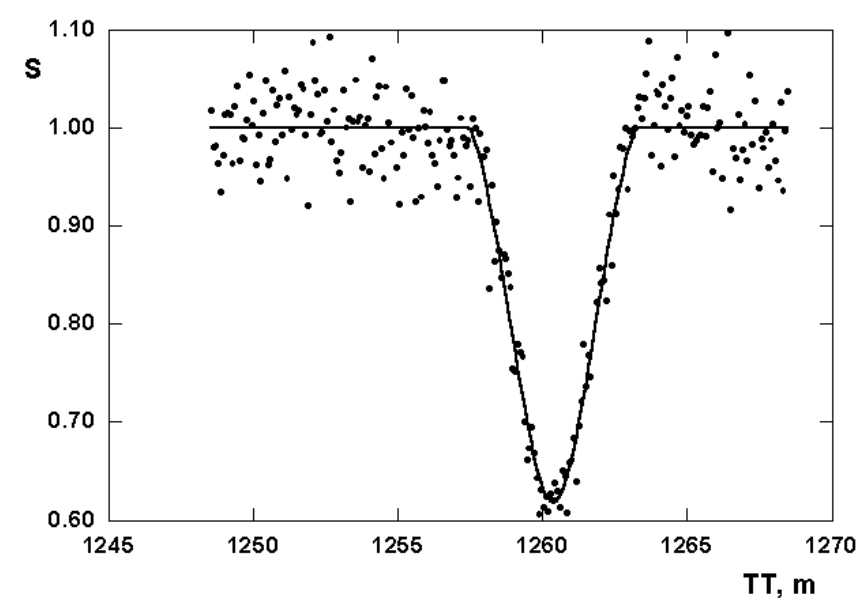

Fig. 8. The fit of the theory to the photometric observations 970719-3O1-a

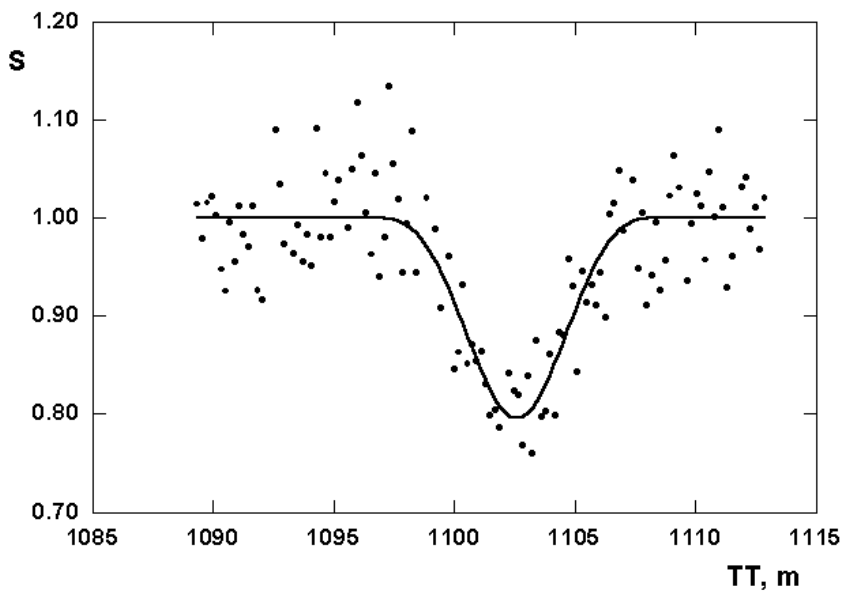

Fig. 9. The fit of the theory to the photometric observations 970724-1E4-a

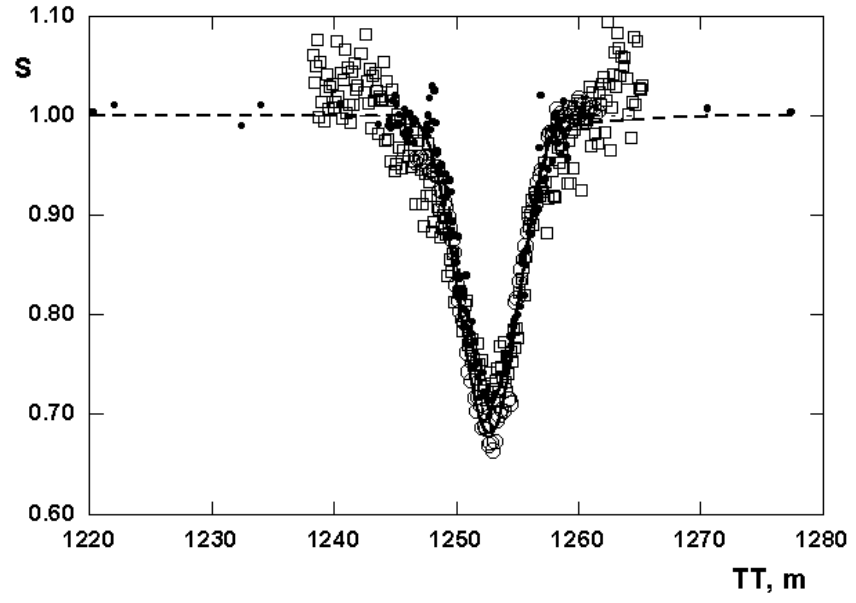

Fig. 10. The fit of the theory to the photometric observations 970725-1E4-a (squares), 970725-1E4-g (dots, dashed line) and 970725-1E4-t (circles)

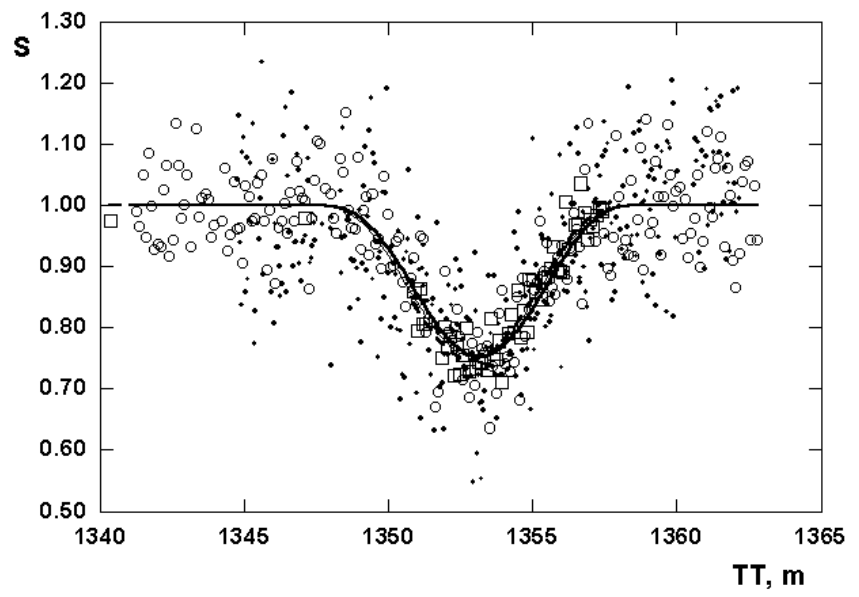

Fig. 11. The fit of the theory to the photometric observations 970725-3E2-a (circles), 970725-3E2-k (dots) and 970725-3E2-g (squares)

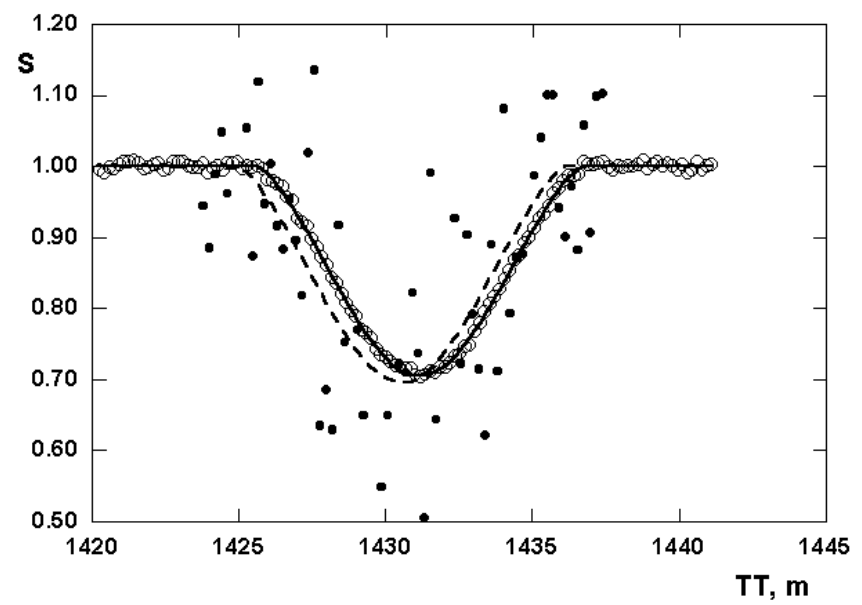

Fig. 12. The fit of the theory to the photometric observations 970725-3O2-k (dots, dashed line) and 970725-3O2-t (circles) 


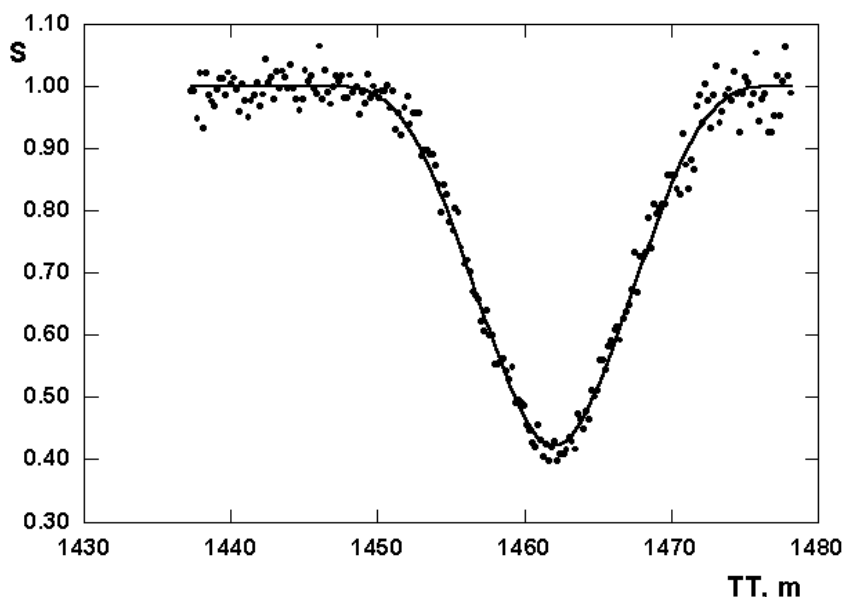

Fig. 13. The fit of the theory to the photometric observations 970731-4E3-e. The time is measured from $0^{\mathrm{h}}$ July 31,1997

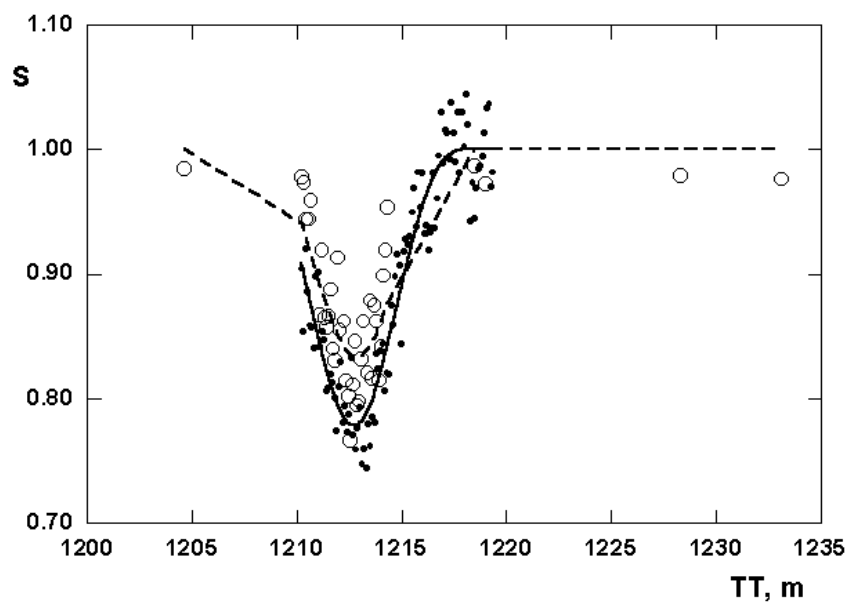

Fig. 14. The fit of the theory to the photometric observations 970801-4E2-g (circles, dashed line) and 970801-4E2-l (dots)

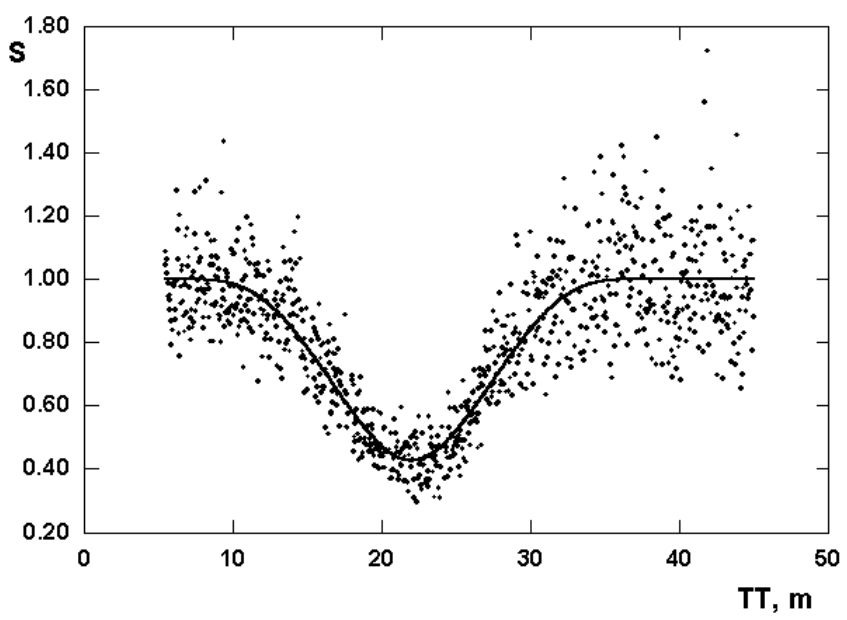

Fig. 15. The fit of the theory to the photometric observations 970801-4E3-k

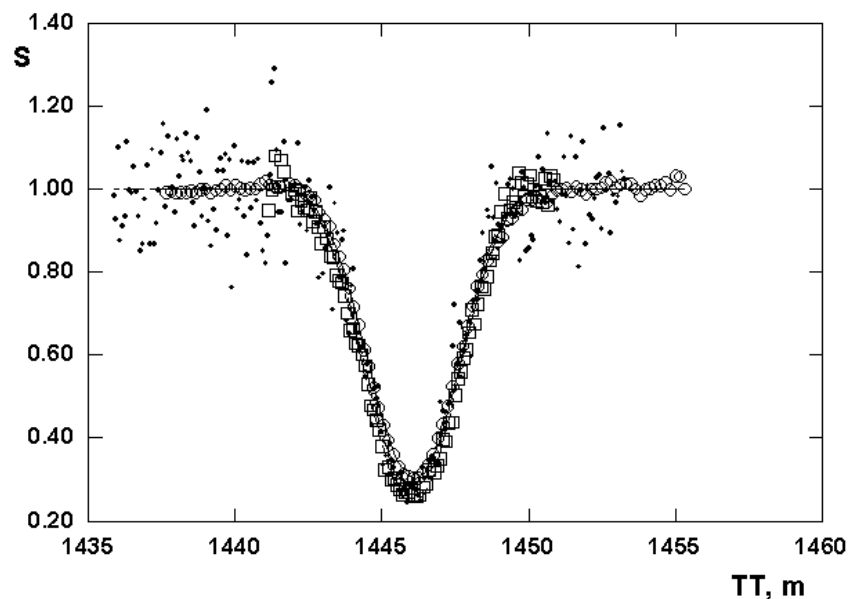

Fig. 16. The fit of the theory to the photometric observations 970803-4E1-k (dots), 970803-4E1-l (squares) and 970803-4E1-t (circles). The time is measured from $0^{\mathrm{h}}$ Aug. 2, 1997

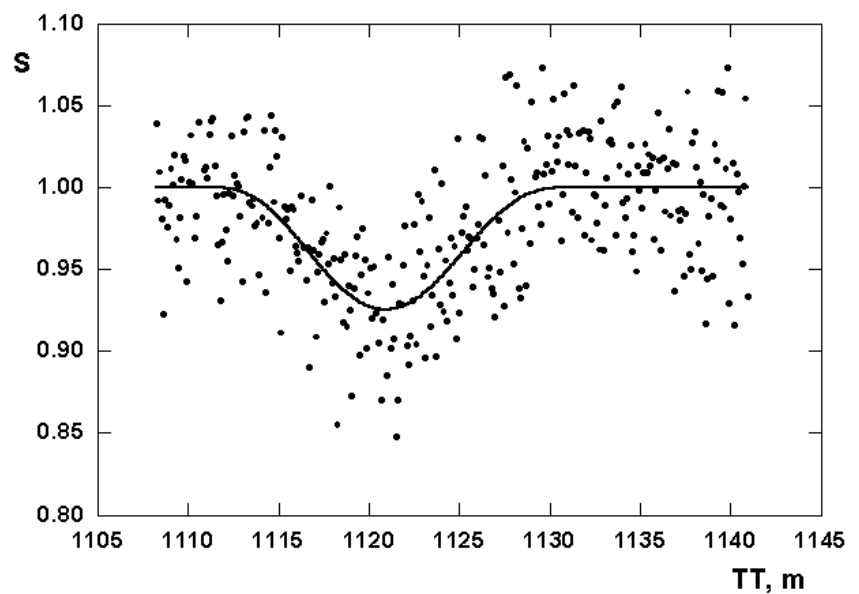

Fig. 17. The fit of the theory to the photometric observations 970830-3E2-a

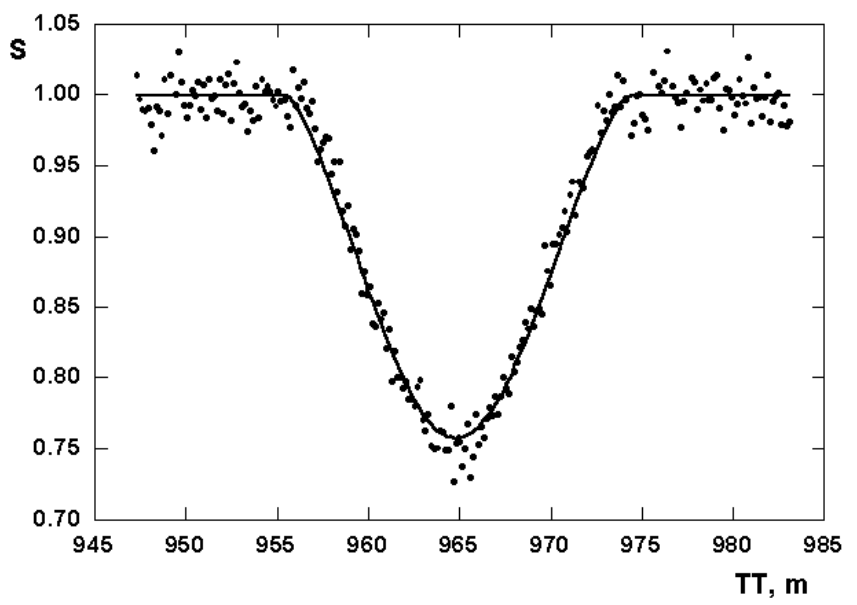

Fig. 18. The fit of the theory to the photometric observations 970830-3O2-a 


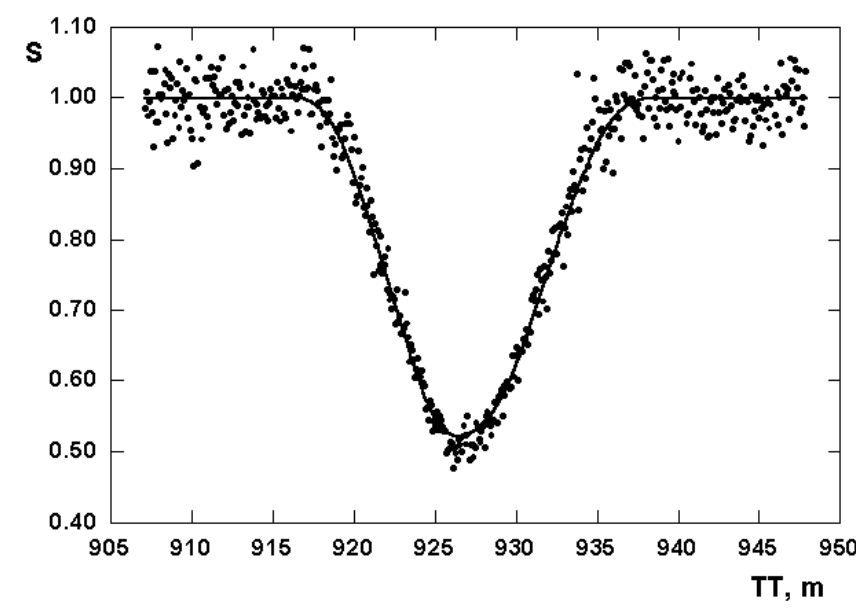

Fig. 19. The fit of the theory to the photometric observations 970903-1E3-a

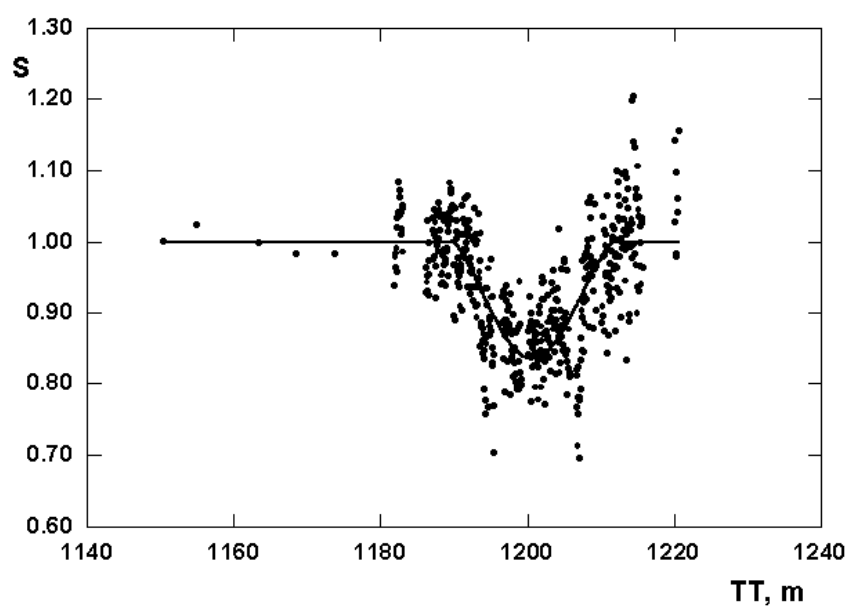

Fig. 20. The fit of the theory to the photometric observations 970906-3O2-g

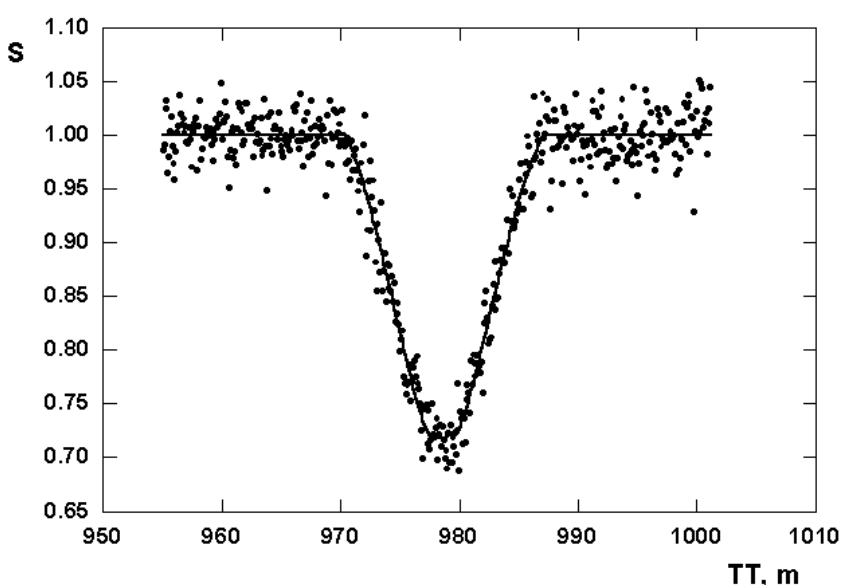

Fig. 21. The fit of the theory to the photometric observations 970910-1O3-a

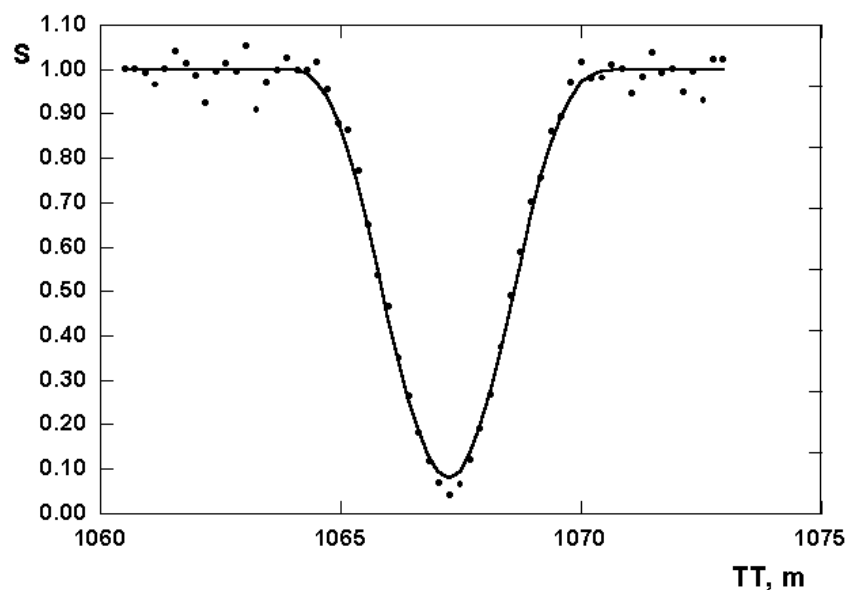

Fig. 22. The fit of the theory to the photometric observations 970914-3E1-e

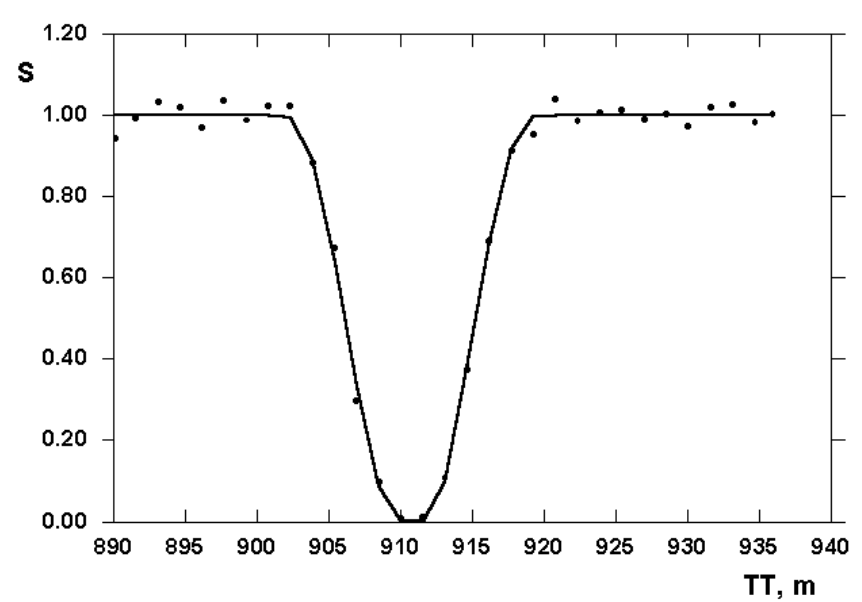

Fig. 23. The fit of the theory to the photometric observations 970915-3E2-a

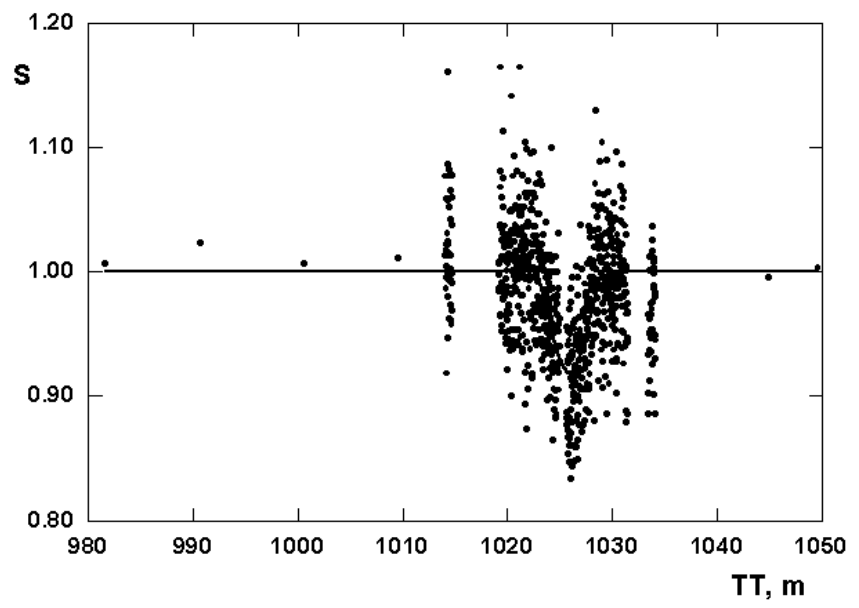

Fig. 24. The fit of the theory to the photometric observations 970918-1O3-g 


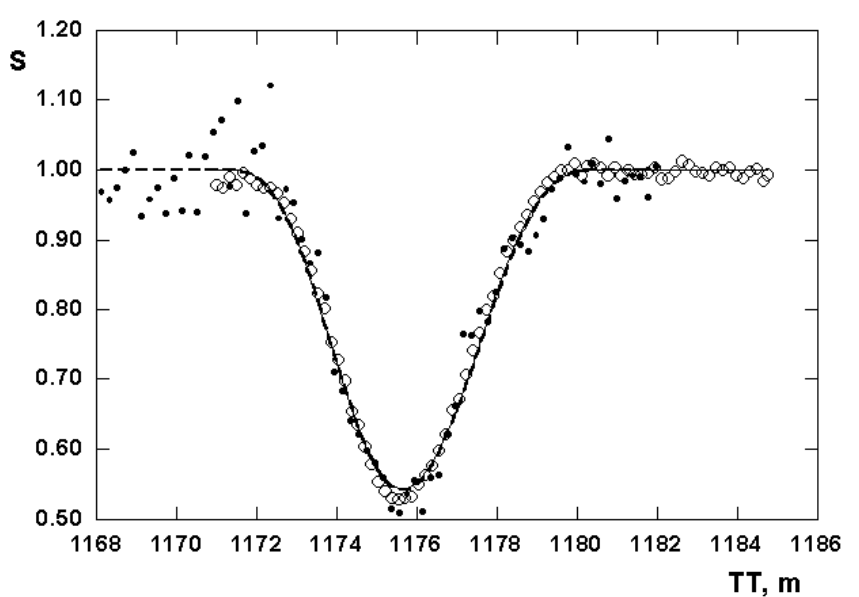

Fig. 25. The fit of the theory to the photometric observations 970918-1E3-e (dots, dashed line) and 970918-1E3-t (circles)

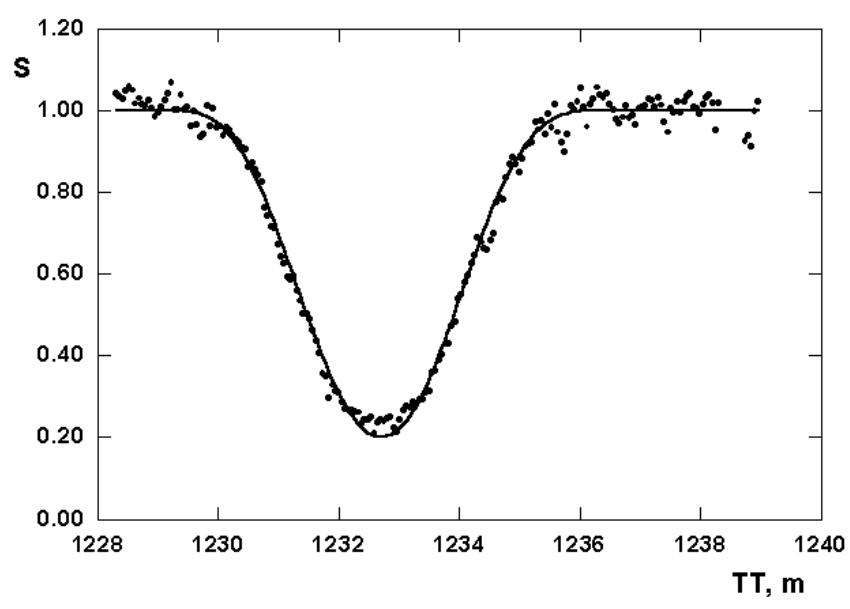

Fig. 26. The fit of the theory to the photometric observations 970921-3E1-1

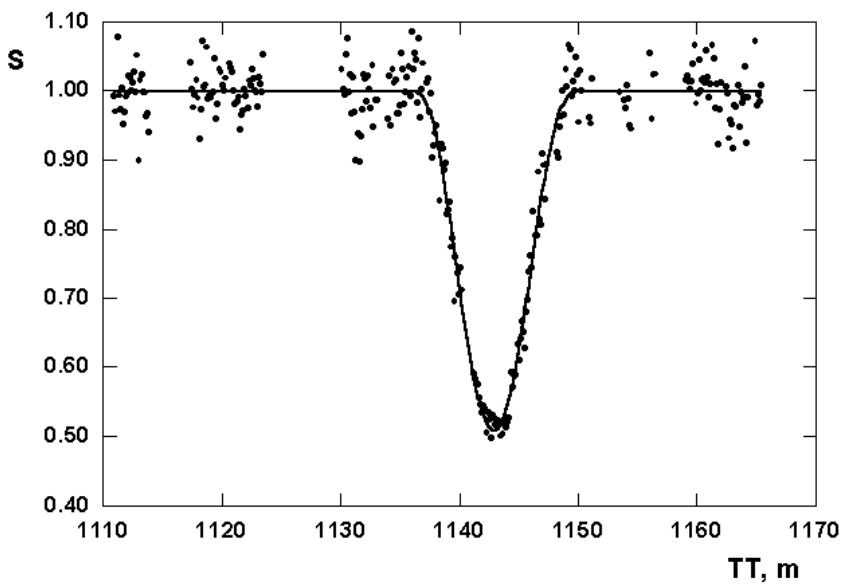

Fig. 27. The fit of the theory to the photometric observations 970922-3E2-g

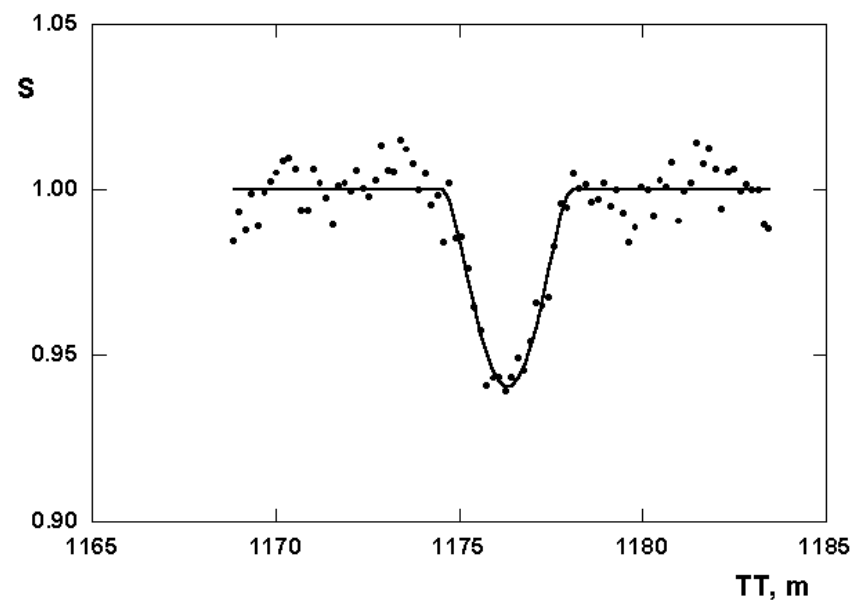

Fig. 28. The fit of the theory to the photometric observations 971007-4O1-t

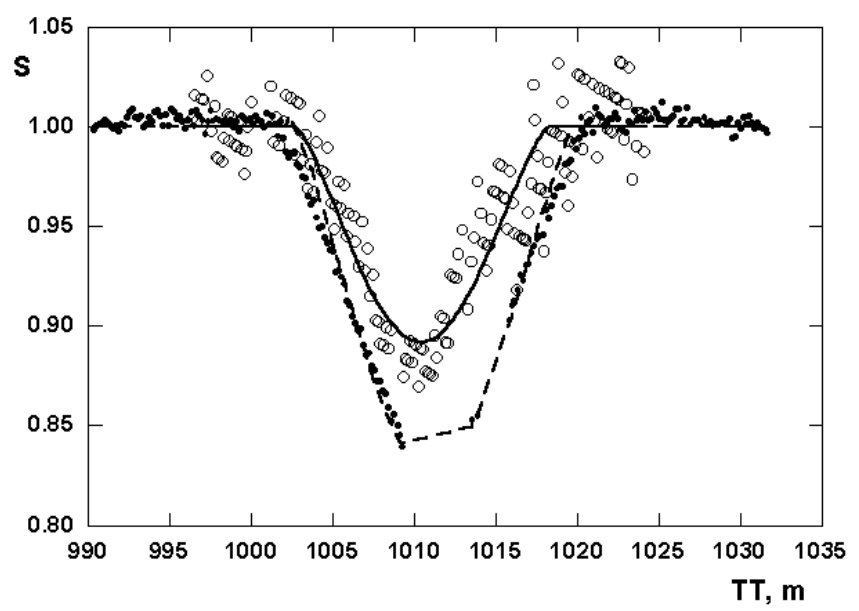

Fig. 29. The fit of the theory to the photometric observations 971109-4O2-t (dots, dashed line), 971109-4O2-w (circles)

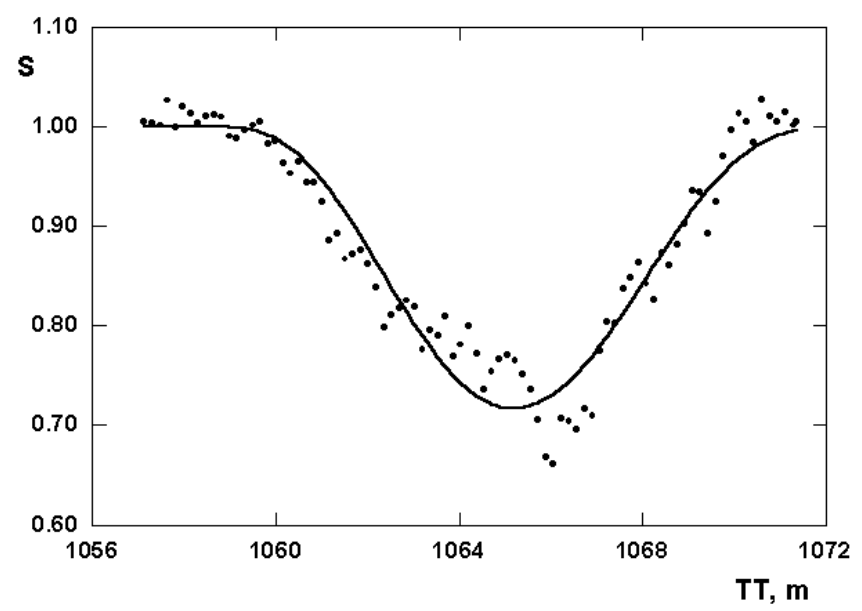

Fig. 30. The fit of the theory to the photometric observations 971110-3E1-t 


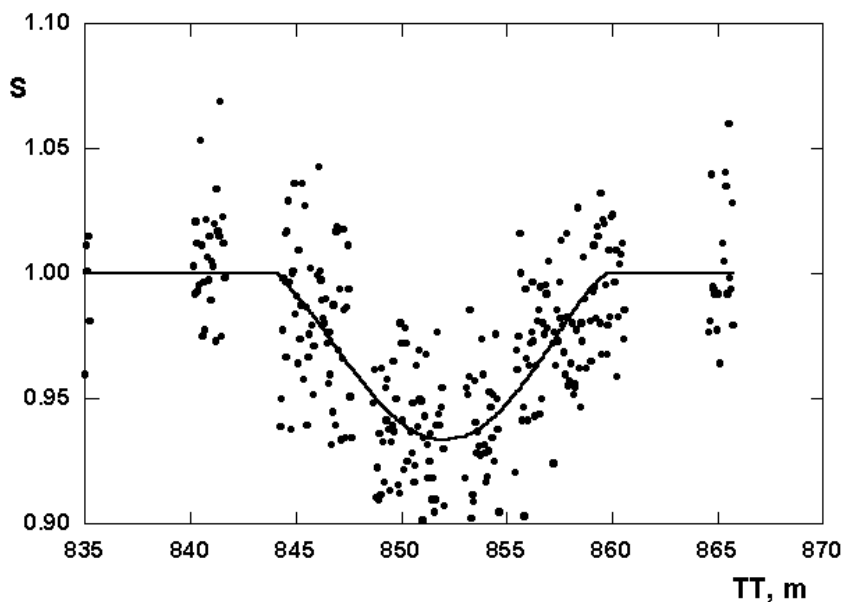

Fig. 31. The fit of the theory to the photometric observations 971118-3O1-g 